\title{
On the $p$-Regularity of Stiefel Manifolds
}

\author{
Dedicated to Professor Shôrô Araki for his 60th birthday
}

By

\author{
Atsushi Yamaguchi*
}

\section{Entroduction}

A topological space which has the homotopy type of a finite $C W$-complex is called $p$-regular if its localization at a prime $p$ is homotopy equivalent to a product of a certain number of spheres localized at $p$. We note that, if $X$ is $p$ regular, the rational cohomology of $X$ determines the number and dimensions of spheres which appear in the decomposition into the product. J.-P. Serre gave, in one of his celebrated papers ([19]), a necessary and sufficient condition for the $p$-regularity of classical Lie groups. For example, $U(n)$ (resp. $S p(n))$ is $p$-regular if and only if $n \leqq p$ (resp. $2 n \leqq p$ ). The purpose of this paper is to generalize the result of Serre to complex and quaternionic Stiefel manifolds. The $p$-regularity of Stiefel manifolds was also studied by Y. Hemmi ([6]) from the $H$-space theoretical point of view. He showed that, for an odd prime $p$, there are infinitely many complex and quaternionic Stiefel manifolds which are $p$-regular (see [6] Theorem 3.3 for details). Our result is somewhat stronger than his in the sense that, for an odd prime $p$, we determine whether a complex (quaternionic) Stiefel manifold is $p$-regular or not except for finitely many undecided cases, and we also deal with the case $p=2$. In particular, we settle the $p$-regularity problem on complex Stiefel manifolds for $p=2$ and 3, and there are still two (resp. six) undecided cases for $p=5$ (resp. $p=7$ ) (see Example 4.10).

Following James ([8]), we denote by $V_{n, k}, W_{n, k}$ and $X_{n, k}$ the real, complex and quaternionic Stiefel manifolds $O(n) / O(n-k), U(n) / U(n-k)$ and $S p(n) / S p(n$ $-k$ ) respectively. For an odd prime $p$, by examining the action of the Steenrod operation $P^{1}$ on $H^{*}\left(W_{n+k, k} ; \mathbf{F}_{p}\right)$ and $H^{*}\left(X_{n+k, k} ; \mathbf{F}_{p}\right)$, we easily derive a necessary condition that the $p$-regularity of $W_{n+k, k}\left(\operatorname{resp} . X_{n+k, k}\right)$ implies $k \leqq p-1$, or $k=p$ and $p \mid n$ (resp. $k \leqq(p-1) / 2$, or $k=(p+1) / 2$ and $p \mid 2 n+1)$.

The main results of this paper are as follows.

Theorem 2.17. $W_{n+2,2}$ is not 2-regular unless $n=0$ or 2 , and $X_{n+2,2}$ is not 2-regular for any $n \geqq 0$.

Communicated by N. Shimada, July 4, 1988.

* Department of Mathematics, University of Osaka Prefecture, Sakai, Osaka 591, Japan. 
We define sets of integers $C_{n+k, k}(\mathbb{F})(\mathbb{F}=\mathbb{C}$ or $\mathbb{H})$ and $\mathbb{I}(n ; p)(\mathrm{p}$ : odd prime) by $C_{n+k, k}(\mathbb{F})=\left\{i \in \mathbb{Z} \mid i=\sum_{j=1}^{k} \varepsilon_{j}(d n+d j-1), \varepsilon_{j}=0\right.$ or 1$\}$, where $d=2$ if $\mathbb{F}=\mathbb{C}$ and $d=4$ if $\mathbb{F}=\mathbb{H}, \mathbb{M}(n ; p)=\left\{i \in \mathbb{Z} \mid i=2 p n+2 j(p-1)\right.$ for $j \geqq 1$, or $i=2\left(r_{n}\right.$ $+1)(p-1)+2 p n-1$ if $\left.r_{n}>0\right\}$, where $r_{n}$ is defined by $-n-1=p q_{n}$ $+r_{n}, 0 \leqq r_{n}<p$. Note that $\mathbb{\Pi}(n ; p)=\{i \in \mathbb{Z} \mid i=2 p n+2 j(p-1)$ for $j \geqq 1\}$ in the case $r_{n}=0$.

Theorem 4.7. i) For $k \leqq p-1, \quad W_{n+k, k}$ is p-regular if $\bigcup_{j=0}^{k-1}$ $\left(C_{n+k, k-j}(\mathbb{C}) \bigcap \mathbb{\Pi}(n+j ; p)\right)$ is empty. In particular, it is p-regular if $n \geqq\left(k^{2}-2 p\right.$ $+3) /(2 p-2 k)$. For $k=p, W_{m p+p, p}$ is $p$-regular if $m \geqq(p-1) / 2$.

ii) For $k \leqq(p-1) / 2, X_{n+k, k}$ is $p$-regular if $\bigcup_{j=0}^{k-1}\left(C_{n+k, k-j}(\mathbb{H}) \bigcap \mathbb{M}(2 n+2 j\right.$ $+1 ; p))$ is empty. In particular, it is p-regular if $n \geqq\left(2 k^{2}+k-4 p+3\right) /(4 p$ $-4 k)$. For $k=p, X_{n+(p+1) / 2,(p+1) / 2}$ is $p$-regular if $p \mid 2 n+1$.

If follows from the above theorem that, for each odd prime $p$, almost all ( = except for finitely many undecided cases) $W_{n+k, k}$ and $X_{n+k, k}$ are $p$-regular if the necessary condition we mentioned above is satisfied. And as a corollary of Theorem 2.17, we show that the attaching maps of the top cells of $W_{n+2,2}$ and $X_{n+2,2}$ produce two families of infinite number of elements of the 2-components of the unstable homotopy groups of spheres, and that the loop space $\Omega W_{n+2,2}$ (resp. $\Omega X_{n+2,2}$ ) has an exotic loop space structure if $n$ is even and greater than two (resp. $n \equiv 6$ modulo 8 ). We also discuss the non-2-regularity of real Stiefel manifolds in the appendix and prove an analogous theorem.

We prove the non-2-regularity of $W_{n+2,2}$ and $X_{n+2,2}$ by showing that the classifying maps of spherical fibrations $S^{2 n+1} \rightarrow W_{n+2,2} \rightarrow S^{2 n+3}$ and $S^{4 n+3}$ $\rightarrow X_{n+2,2} \rightarrow S^{4 n+7}$ are non-trivial. For this end, we examine the $J$ homomorphism in the unstable range. On the other hand, we prove the $p$ regularity for odd prime $p$ under the condition of the above theorem by showing that spherical fibrations $S^{2 n+2 j+1} \rightarrow W_{n+k, k-j} \rightarrow W_{n+k, k-j-1}$ and $S^{4 n+4 j+3}$ $\rightarrow X_{n+k, k-j} \rightarrow X_{n+k, k-j-1}$ are fiber homotopy equivalent to product fibrations for $j=0,1, \ldots, k-1$. To show this, we demonstrate the existence of left homotopy inverses of the canonical inclusions $S^{2 n+2 j+1} \rightarrow W_{n+k, k-j}$ and $S^{4 n+4 j+3} \rightarrow X_{n+k, k-j}$ by using obstruction theory.

In Section 1, we give a necessary and sufficient condition for the splitting of a quasiprojective space into a wedge of spheres at a prime $p$. In fact, we prove the following three conditions are equivalent; a) $k \leqq p-1$, or $k=p$ and $p \mid n$. b) $Q_{n+k, k}(\mathbb{C})=\Sigma\left(\mathbb{C P}^{n+k-1} / \mathbb{C P}^{n-1}\right)$ is homotopy equivalent to $\vee_{i=1}^{k} S^{2 n+2 i-1}$ at prime $p$. c) $\Omega W_{n+k, k}$ is homotopy equivalent to $\Omega\left(\prod_{i=1}^{k} S^{2 n+2 i-1}\right)$ at prime p. A similar result is also obtained for the quaternionic case. In Section 2, we discuss the non-2-regularity of $W_{n+2,2}$ and $X_{n+2,2}$. We reduce the problem on the unstable $J$-homomorphism, which is examined by applying theorems of Toda ([21], Theorem 11.7) and Mahowald-Milgram ([10] Theorem A, [14] Theorem $8.5,8.21$ ). Section 3 is a preparation for Section 4 . We compute some $p$ - 
localized homotopy groups of the homotopy fiber of the natural inclusion $S^{2 n+1}$ $\rightarrow Q S^{2 n+1}$. We apply the Adams-Novikov spectral sequence based on the BPhomology theory to the computation, and determine the necessary and sufficient condition for the vanishing of "obstruction groups" $\pi_{i}\left(Q S^{2 n+1}, S^{2 n+1}\right)$ for sufficiently many $i$ 's. In Section 4, we first show the existence of maps $f:\left(W_{n+k, k}, S^{2 n+1}\right) \rightarrow\left(Q S^{2 n+1}, S^{2 n+1}\right) \quad$ and $\quad g:\left(X_{n+k, k}, S^{4 n+3}\right) \rightarrow\left(Q S^{4 n+3}, S^{4 n+3}\right)$ such that the restrictions to $S^{2 n+1}$ and $S^{4 n+3}$ are maps of degree 1 if $n$ and $k$ satisfy the necessary condition. And by applying obstruction theory, we see that these maps can be deformed to left homotopy inverses of the canonical inclusions. We prove in the appendix that the real Stiefel manifold $V_{n+2,2}$ is not 2-regular unless $n=0,2$ or 6 .

The author would like to thank Jeff Smith, Jack Morava, Steve Wilson and Masana Harada for invaluable conversations, and thank Jean-Pierre Meyer for informing him of R.J. Milgram's work [14]. And he wishes to express his gratitude to all the faculty and staff of the Mathematics Department of University of Osaka Prefecture for their support to him during his visit to the Johns Hopkins University.

\section{Notations and Terminology}

When we use the term "at prime $p$ " in a statement, this means that the statement is valid if we localize spaces and groups in the statement. We denote the mod $p$ homology (resp. cohomology) of $X$ by $H_{*}(X)\left(\right.$ resp. $\left.H^{*}(X)\right)$ when the prime $p$ is clear from the context. Otherwise, we denote by $H_{*}(X ; R)$ (resp. $H^{*}(X ; R)$ ) the homology (resp. cohomology) of $X$ with coefficients in $R$. We list some notations which are frequently used in this paper.

$V_{n, k}, W_{n, k}$ and $X_{n, k}$ : the real, complex and quaternionic Stiefel manifolds $O(n) / O(n-k), U(n) / U(n-k)$ and $S p(n) / S p(n-k)$ respectively.

$R\left\{x_{1}, x_{2}, \ldots\right\}$ : the free $R$-module generated by $x_{1}, x_{2}, \ldots$.

$\mathbb{F}_{p}\left[x_{1}, x_{2}, \ldots\right]$ : the polynomial algebra over $\mathbb{F}_{p}$ generated by $x_{1}, x_{2}, \ldots$.

$E\left(x_{1}, x_{2}, \ldots\right)$ : the exterior algebra over $\mathbf{F}_{p}$ generated by $x_{1}, x_{2}, \ldots$

$M_{(p)}$ : the localization at prime $p$ of an abelian group $M$.

$\left(\begin{array}{l}n \\ k\end{array}\right)$ : the binomial coefficient $n(n-1) \ldots .(n-k+1) / k$ !

$[x]$ : the Gauss symbol of $x \in \mathbb{R}$, that is, the largest integer that does not exceed $x$.

$k \mid n(k, n \in \mathbb{Z}) ; k$ divides $n$.

$Q_{n, k}(\mathbf{F})$ : the quasiprojective space over field $\mathbf{F}(\mathbf{F}=\mathbb{R}, \mathbf{C}$ or $\mathbb{H})$.

$\mathbb{P}_{n}^{m}$ : the stunted real projective space $\mathbf{R} \mathbf{P}^{m} / \mathbf{R} \mathbb{P}^{n-1}$.

$F(n)$ : the homotopy fiber of the natural inclusion $S^{n} \rightarrow Q S^{n}$.

$\pi_{r}^{S}(X)$ : the stable homotopy group of a space (spectrum) $X$. 


\section{$\S \mathbb{1}$. Splitting of Quasiprojective Spaces}

Let $Q_{n, k}(\mathbb{F})$ be the quasiprojective space over a field $\mathbb{F}(\mathbb{F}=\mathbb{R}, \mathbb{C}$, or $\mathbb{H})$ defined in [7](see also [8], [20]). We identify $Q_{n, k}(\mathbb{R}), Q_{n, k}(\mathbb{C})$ and $Q_{n, k}(\mathbb{H})$ with subcomplexes of $V_{n, k}, W_{n, k}$ and $X_{n, k}$ respectively, then the pair of $C W$-complexes $\left(W_{n+k, k}, Q_{n+k, k}(\mathbb{C})\right)\left(\right.$ resp. $\left.\quad\left(X_{n+k, k}, Q_{n+k, k}(\mathbb{H})\right)\right)$ is $(4 n+3)$-connected (resp. $(8 n$ $+9)$-connected), and the pair $\left(V_{n+k, k}, Q_{n+k, k}(\mathbf{R})\right)$ is $2 n$-connected at prime 2. Thus quasiprojective spaces are approximations of Stiefel manifolds in the stable range. The mod $p$ cohomology of $Q_{n+k, k}(\mathbb{F})(\mathbb{F}=\mathbb{C}$ or $\mathbb{H})$ and the action of the Steenrod operations are given by the following.

Lemma 1.1. i) $\widetilde{H}^{*}\left(Q_{n+k, k}(\mathbb{C})\right)=\mathbb{F}_{p}\left\{x_{2 n+1}, x_{2 n+3}, \ldots, x_{2 n+2 i-1}, \ldots, x_{2 n+2 k-1}\right\}$, $\operatorname{deg} x_{i}=i . \quad P^{s} x_{2 n+2 i-1}=\left(\begin{array}{c}n+i-1 \\ s\end{array}\right) x_{2 n+2 i+2 s(p-1)-1}$ (We put $P^{s}=S q^{2 s}$ if $p$ $=2)$.

ii) $\tilde{H}^{*}\left(Q_{n+k, k}(\mathbf{H})\right)=\mathbb{F}_{p}\left\{x_{4 n+3}, \quad x_{4 n+7}, \ldots, \quad x_{4 n+4 i-1}, \ldots, x_{4 n+4 k-1}\right\}, \operatorname{deg} x_{i}=i$. $P^{s} x_{4 n+4 i-1}=\left(\begin{array}{c}2 n+2 i-1 \\ s\end{array}\right) x_{4 n+4 i+2 s(p-1)-1}$ (If $p=2$, we put $P^{s}=S q^{2 s}$ as above, and $x_{i}=0$ if $i \neq 3$ modulo 4$)$.

Proof. Since $Q_{n+k, k}(\mathbb{C})$ is a suspension of a stunted complex projective space $\mathbb{C P}^{n+k-1} / \mathbb{C P}^{n-1}$, i) follows from the action of $P^{s}$ on $H^{*}\left(\mathbb{C P}^{\infty}\right)([20]$, Chap. I, VI). For ii), there is a map $c^{\prime}: Q_{n+k, k}(\mathbb{H}) \rightarrow Q_{2 n+2 k, 2 k}(\mathbb{C})$ induced by the canonical inclusion $S p(n) \rightarrow U(2 n)$ and $c^{\prime *}: \tilde{H}^{*}\left(Q_{2 n+2 k, 2 k}(\mathbb{C})\right) \rightarrow \widetilde{H}^{*}\left(Q_{n+k, k}(\mathbb{H})\right)$ maps $x_{4 n+4 i-1}$ to $x_{4 n+4 i-1}$, and $x_{4 n+4 i-3}$ to zero. Hence ii) follows from i).

In particular, we have $P^{1} x_{2 n+2 i-1}=(n+i-1) x_{2 n+2(i+p-1)-1}$ in $\tilde{H}^{*}\left(Q_{n+k, k}(\mathbb{C})\right)$ and $P^{1} x_{4 n+4 i-1}=(2 n+2 i-1) x_{4 n+4 i+2(p-1)-1}$ for $p>2$, $S q^{4} x_{4 n+4 i-1}=(n+i-1) x_{4 n+4 i+3}$ for $p=2$ in $\widetilde{H}_{*}\left(Q_{n+k, k}(\mathbb{H})\right)$. Therefore

Corollary 1.2. The action of the Steenrod operations on $\tilde{H}^{*}\left(Q_{n+k, k}(\mathbb{F})\right)$ is trivial in the following cases and non-trivial otherwise;

For $\mathbb{F}=\mathbb{C}, k \leqq p-1$, or $k=p$ and $p \mid n$.

For $\mathbb{E}=\mathbb{H}, k \leqq(p-1) / 2$, or $k=(p+1) / 2$ and $p \mid 2 n+1$ if $p>2$. In the case $p$ $=2, k=1$, or $k=2$ and $2 \mid n$.

We consider the case $p=k=2$ first. Let $\Delta_{C}: \pi_{2 n+3}\left(S^{2 n+3}\right) \rightarrow \pi_{2 n+2}\left(S^{2 n+1}\right)$ and $\Delta_{H}: \pi_{4 n+7}\left(S^{4 n+7}\right) \rightarrow \pi_{4 n+6}\left(S^{4 n+3}\right)$ be the connecting homomorphisms of the long exact sequence associated with the spherical fibrations $S^{2 n+1} \rightarrow W_{n+2,2}$ $\rightarrow S^{2 n+3}, S^{4 n+3} \rightarrow X_{n+2,2} \rightarrow S^{4 n+7}$ respectively. We denote by $l_{n}, \eta_{n}, v^{\prime}, v_{n}$ the generators of $\pi_{n}\left(S^{n}\right), \pi_{n+1}\left(S^{n}\right), \pi_{6}\left(S^{3}\right)$ and $\pi_{n+3}\left(S^{n}\right)(n \geqq 4)$ respectively. Then $Q_{n+2,2}(\mathbb{C})$ and $Q_{n+2,2}(\mathbb{H})$ are cofibers of maps $\Delta_{C} l_{2 n+3}: S^{2 n+2} \rightarrow S^{2 n+1}$ and $\Delta_{H} l_{2 n+7}: S^{4 n+6} \rightarrow S^{4 n+3}$. Since $S q^{2}$ determines whether $\Delta_{C} l_{2 n+3}$ is trivial or not, we have 
Theorem 1.3. $Q_{n+2,2}(\mathrm{C})$ is homotopy equivalent to $S^{2 n+1} \vee S^{2 n+3}$ at prime 2 if and only if $n$ is even. And if $n$ is even, $Q_{n+2,2}(\mathbf{C})$ is (globally) homotopy equivalent to $S^{2 n+1} \vee S^{2 n+3}$.

In the quaternionic case, the condition of Corollary 1.2 does not necessarily imply the splitting of $Q_{n+2,2}(\mathrm{H})$ at prime 2, because $S q^{4}$ cannot detect all elements of $\pi_{4 n+6}\left(S^{4 n+3}\right)$. We need the following result of James.

Theorem $1.4([7],[8]) . \quad \Delta_{H^{l} l_{n+7}}= \begin{cases}v^{\prime} & \text { for } n=0 \\ (n+2) v_{4 n+3} & \text { for } n \geqq 1\end{cases}$

Since $v_{4 n+3}$ has order 24 , the above immediately implies

Theorem 1.5. $Q_{n+2,2}(\mathbf{H})$ is homotopy equivalent to $S^{4 n+3} \vee S^{4 n+7}$ at prime 2 if and only if $n \equiv 6$ modulo 8 , and it is homotopy equivalent to $S^{4 n+3} \vee S^{4 n+7}$ at prime 3 if and only if $n \equiv 1$ modulo 3 . Moreover, if $n \equiv 22$ modulo $24, Q_{n+2,2}(\mathbf{H})$ is globally homotopy equivalent to $S^{4 n+3} \vee S^{4 n+7}$.

And also Theorem 1.4 implies

Theorem 1.6. $X_{n+2,2}$ is not 2-regular if $n \neq 1$ modulo 8 .

Proof. In fact, if $X_{n+2,2}$ is 2-regular, $\pi_{4 n+6}\left(X_{n+2,2}\right)_{(2)} \cong \pi_{4 n+6}\left(S^{4 n+3} \times S^{4 n+7}\right)_{(2)}$ $\cong \mathbf{Z} / 8$. On the other hand, $\pi_{4 n+6}\left(X_{n+2,2}\right)_{(2)} \cong\left(\pi_{4 n+6}\left(S^{4 n+3}\right) / \operatorname{Im} \Delta_{H}\right)_{(2)} \cong \mathbf{Z} /(8, n+2)$ which is not isomorphic to $\mathbf{Z} / 8$ if $n \neq 6$ modulo 8 .

We therefore consider the following conditions $(\mathrm{C}),(\mathrm{H})$ on $p, k, n$ instead of those of Corollary 1.2.

(C) $k \leqq p-1$, or $k=p$ and $p \mid n$

(H) $k \leqq(p-1) / 2$, or $k=(p+1) / 2$ and $p \mid 2 n+1$ if $p>2$, $k=2$ and $n \equiv 6$ modulo 8 if $p=2$.

Lemma 1.7. Let $p$ be an odd prime and let $X$ be a space of finite type. Suppose that $X$ is $2 n$-connected at prime $p$ and $H_{2 n+2 i}\left(X ; \mathbf{F}_{p}\right)=0$ for $i<p-1$, then $\pi_{2 n+2 i}(X)_{(p)}=0$ for $i<p-1$.

Proof. We may assme that $\pi_{2 n+j}(X)_{(p)} \neq 0$ for some $j<2(p-1)$, otherwise there is nothing to prove. Then, by the assumption, $\min \left\{j \mid \pi_{2 n+j}(X)_{(p)} \neq 0\right\}$ is odd, and we define an integer $i(X)$ by $2 i(X)+1=\min \left\{j \mid \pi_{2 n+j}(X)_{(p)} \neq 0\right\}$. We prove the assertion by induction on $l=p-2-i(X)$. First we note that $l \geqq 0$ by the above assumption and that the assertion holds if $l=0$. Considering the universal coefficient theorem, it follows from the assumption that $H_{2 n+2 i+1}(X$; $\left.\mathbb{Z}_{(p)}\right)$ is torsion free for $i<p-2$. Hence so is the lowest non-trivial homotopy 
group $\pi_{2 n+2 i(X)+1}(X)_{(p)}$. Applying the killing method to $X$, we have a fibering $X^{\prime} \rightarrow X \rightarrow K\left(\mathbb{Z}^{r}, 2 n+2 i(X)+1\right)\left(r=\right.$ the rank of $\left.\pi_{2 n+2 i(X)+1}(X)_{(p)}\right)$ such that $f_{*}$ : $\pi_{2 n+2 i(X)+1}(X)_{(p)} \rightarrow \pi_{2 n+2 i(X)+1}\left(K\left(\mathbb{Z}^{r}, 2 n+2 i(X)+1\right)\right)_{(p)}$ is an isomorphism. Then, the standard argument on the Serre spectral sequence associated with this fibering shows that $X^{\prime}$ is $(2 n+2 i(X)+2)$-connected at prime $p$ and $H_{2 n+2 j}\left(X^{\prime}\right.$; $\left.\mathbb{F}_{p}\right)=0$ for $j<p-1$. Thus $i\left(X^{\prime}\right)>i(X)$ and $p-2-i\left(X^{\prime}\right)<l$, and the inductive assuption applies to $X^{\prime}$.

Let $f_{n, i}: S^{2 n+2 i-2} \rightarrow Q_{n+i-1, i-1}(\mathbb{C})$ and $g_{n, i}: S^{4 n+4 i-2} \rightarrow Q_{n+i-1, i-1}(\mathbb{H})$ be the attaching maps of $(2 n+2 i-1)$-cell and $(4 n+4 i-1)$-cell. Note that quasiprojective spaces $Q_{n+i-1, i-1}(\mathbb{C})$ and $Q_{n+i-1, i-1}(\mathrm{H})$ satisfy the assumptions of the preceding lemma. It follows that $f_{n, i} \simeq 0$ if $i<p$ and $g_{n, i} \simeq 0$ if $i<(p+1) / 2$, when we localize spaces at prime $p$.

Theorem 1.8. Let $p$ be an odd prime. $Q_{n+k, k}(\mathbb{C})$ is homotopy equivalent to $\vee_{i=1}^{k} S^{2 n+2 i-1}$ at prime $p$ if $k<p$, or $k=p$ and $p \mid n$. $Q_{n+k, k}(\mathrm{H})$ is homotopy equivalent to $\vee_{i=1}^{k} S^{4 n+4 i-1}$ at prime $p$ if $k<(p+1) / 2$, or $k=(p+1) / 2$ and $p \mid 2 n+1$.

Proof. In the case $\mathbb{F}=\mathbb{C}, k<p$ or $\mathbb{F}=\mathbb{H}, k<(p+1) / 2$, the assertion follows by induction of $k$ since attaching maps are all null homotopic at prime p. We consider the case $\mathbb{F}=\mathbb{C}, k=p$ and $p \mid n$. If $n=0, Q_{p, p}(\mathbb{C})=\Sigma\left(\mathbb{C P}^{p-1}{ }_{+}\right)$ which is homotopy equivalent to $S^{1} \vee \Sigma \mathbb{C P}^{p-1}$. Since $\Sigma \mathbb{C P}^{i-1}$ is 2-connected and satisfies the assumptions of Lemma 1.7, it follows that each attaching map $S^{2 i} \rightarrow \Sigma \mathbb{C P}^{i-1}$ is null homotopic at prime $p$ if $i<p$. Hence $\Sigma \mathbb{C} \mathbb{P}^{p-1}$ is homotopy equivalent to $\vee_{i=2}^{p} S^{2 i-1}$ at prime $p$. If $n>0$, then $n \geqq p$ since $p \mid n$. Hence homotopy group $\pi_{2 n+2 p-2}\left(Q_{n+p-1, p-1}(\mathbb{C})\right)$ is in the stable range. By the homotopy equivalence $Q_{n+p-1, p-1}(\mathbb{C}) \simeq \bigvee_{i=1}^{p-1} S^{2 n+2 i-1}$ at prime $p$, we have $\pi_{2 n+2 p-2}\left(Q_{n+p-1, p-1}(\mathbb{C})\right)_{(p)} \cong \sum_{i=1}^{p-1} \pi_{2 n+2 p-2}\left(S^{2 n+2 i-1}\right)_{(p)}=\pi_{2 n+2 p-2}$ $\left(S^{2 n+1}\right)_{(p)}(\cong \mathbb{Z} / p)$, which implies that attaching map $f_{n, p}$ can be factorized as $S^{2 n+2 p-2} \stackrel{f^{\prime}}{\longrightarrow} S^{2 n+1}=Q_{n+1,1}(\mathbb{C}) \rightarrow Q_{n+p-1, p-1}(\mathbb{C})$ at prime $p$. Let $C_{f}$, be the cofiber of $f^{\prime}$, then we have the following homotopy commutative diagram whose horizontal rows are cofiberings

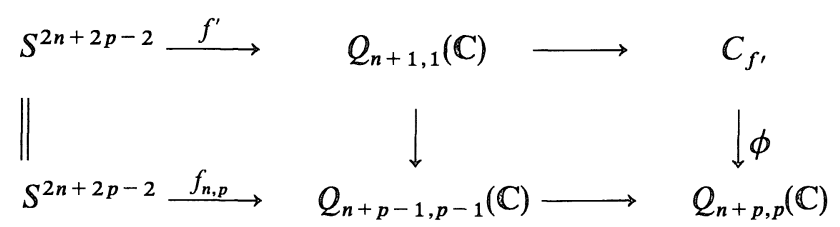

$\phi^{*}: H^{s}\left(Q_{n+p, p}(\mathbb{C}) ; \mathbb{F}_{p}\right) \rightarrow H^{s}\left(C_{f} ; \mathbb{F}_{p}\right)$ is an isomorphism for $s=2 n+1,2 n+2 p$ -1. Thus $P^{1}$ acts on $H^{*}\left(C_{f} ; \mathbb{F}_{p}\right)$ trivially by Corollary 1.2 . It follows $f^{\prime} \simeq 0$, hence $f_{n, p} \simeq 0$ at prime $p$. This proves the assertion in the case $\mathbb{F}=\mathbb{C}, k$ $=p$. Noting that the assumption $p \mid 2 n+1$ implies that 
$\pi_{4 n+2 p}\left(Q_{n+(p-1) / 2,(p-1) / 2}(\mathrm{H})\right)$ is in the stable range, we can prove the assertion similarly in the case $\mathbf{F}=\mathbf{H}, k=(p+1) / 2$ and $p \mid 2 n+1$.

Remark 1.9. If the condition $(\mathrm{C})$ or $(\mathrm{H})$ is not satisfied, it follows from Corollary 1.2 and Theorem 1.4 that neither $Q_{n+k, \mathrm{k}}(\mathbf{C})$ nor $Q_{n+k, k}(\mathbf{H})$ splits into a wedge of spheres at prime $p$, even stably.

After localizing at prime $p$ (if necessary), we have maps $\phi_{C}$ : $\bigvee_{i=1}^{k} S^{2 n+2 i-1} \stackrel{\simeq}{\longrightarrow} Q_{n+k, k}(\mathbf{C}) \rightarrow W_{n+k, k}$ and $\phi_{H}: \bigvee_{i=1}^{k} S^{4 n+4 i-1} \stackrel{\simeq}{\longrightarrow} Q_{n+k, k}(\mathbf{H})$ $\rightarrow X_{n+k, k}$ for $n, k$ satisfying $(\mathrm{C}),(\mathrm{H})$ in each case. Using the loop space structures of $\Omega W_{n+k, k}$ and $\Omega X_{n+k, k}$, we can extend the compositions $\bigvee_{i=1}^{k} \Omega S^{2 n+2 i-1}$

$\rightarrow \Omega\left(\bigvee_{i=1}^{k} S^{2 n+2 i-1}\right) \stackrel{\Omega \phi_{C}}{\longrightarrow} \Omega W_{n+k, k}$ and $\bigvee_{i=1}^{k} \Omega S^{4 n+4 i-1} \rightarrow \Omega\left(\bigvee_{i=1}^{k} S^{4 n+4 i-1}\right)$ $\stackrel{\Omega \phi_{H}}{\longrightarrow} \Omega X_{n+k, k} \quad$ to $\operatorname{maps} \bar{\phi}_{C}: \prod_{i=1}^{k} \Omega S^{2 n+2 i-1} \rightarrow \Omega W_{n+k, k}$ and $\bar{\phi}_{H}$ : $\prod_{i=1}^{k} \Omega S^{4 n+4 i-1} \rightarrow \Omega X_{n+k, k}$. Since the Pontrijagin rings $H_{*}\left(\Omega W_{n+k, k}\right)$ and $H_{*}\left(\Omega X_{n+k, k}\right)$ are commutative, $\bar{\phi}_{C}$ and $\bar{\phi}_{H}$ induce algebra homomorphisms between homologies. By the construction of $\bar{\phi}_{C}$ and $\bar{\phi}_{H} \bar{\phi}_{C *}$ : $\bigotimes_{i=1}^{k} H_{*}\left(\Omega S^{2 n+2 i-1}\right) \rightarrow H_{*}\left(\Omega W_{2 n+k, k}\right) \quad$ and $\quad \bar{\phi}_{H *}: \bigotimes_{i=1}^{k} H_{*}\left(\Omega S^{2 n+4 i-1}\right)$ $\rightarrow H_{*}\left(\Omega X_{n+k, k}\right)$ map each polynomial generator of $H_{*}\left(\Omega S^{2 n+2 i-1}\right)$ and $H_{*}\left(\Omega S^{4 n+4 i-1}\right)$ to a polynomial generator of $H_{*}\left(\Omega W_{n+k, k}\right)$ and $H_{*}\left(\Omega X_{n+k, k}\right)$ respectively. Thus $\bar{\phi}_{C *}$ and $\bar{\phi}_{H *}$ are isomorphisms, and hence they are homotopy equivalences.

Proposition 1.10. i) $\Omega W_{n+k, k}$ is homotopy equivalent to $\prod_{i=1}^{k} \Omega S^{2 n+2 i-1}$ at prime $p$ if and only if the condition (C) is satisfied. And $\Omega W_{n+2,2}$ is globally homotopy equivalent to $\Omega S^{2 n+1} \times \Omega S^{2 n+3}$ if $2 \mid n$.

ii) $\Omega X_{n+k, k}$ is homotopy equivalent to $\prod_{i=1}^{k} \Omega S^{4 n+4 i-1}$ at prime $p$ if and only if the condition $(\mathrm{H})$ is satisfied. And $\Omega X_{n+2,2}$ is globally homotopy equivalent to $\Omega S^{4 n+3} \times \Omega S^{4 n+7}$ if $n \equiv 22$ modulo 24 .

Proof. We already showed the homotopy equivalence if $(\mathrm{C})$ or $(\mathrm{H})$ is satisfied. The converse is easily proved by examining the action of the Steenrod operations on $H_{*}\left(\Omega W_{n+k, k}\right)$ and $H_{*}\left(\Omega X_{n+k, k}\right)$ and by comparing the homotopy group $\pi_{4 n+5}\left(\Omega X_{n+2,2}\right)_{(2)}$ with $\pi_{4 n+5}\left(\Omega S^{4 n+3} \times \Omega S^{4 n+7}\right)_{(2)}$ (See the proof of Theorem 1.6).

Corollary 1.11. $\pi_{*}\left(W_{n+k, k}\right)_{(p)}$ is isomorphic to $\pi_{*}\left(\prod_{i=1}^{k} S^{2 n+2 i-1}\right)_{(p)}$ if $(\mathrm{C})$ is satisfied and $\pi_{*}\left(X_{n+k, k}\right)_{(p)}$ is isomorphic to $\pi_{*}\left(\prod_{i=1}^{k} S^{4 n+4 i-1}\right)_{(p)}$ if $(\mathrm{H})$ is satisfied.

Thus the homotopy groups cannot distinguish $W_{n+k, k}$ from $\prod_{i=1}^{k} S^{2 n+2 i-1}$, nor $X_{n+k, k}$ from $\prod_{i=1}^{k} S^{4 n+4 i-1}$ for $n, k$ satisfying (C) and (H) respectively, if we localize these spaces at prime $p$. 
§2. The Metastable $\mathscr{H}$-Homomorphism and the Nom-2-Regularity We first note that Corollary 1.2 implies,

Proposition 2.1. Neither $W_{n+k, k}$ nor $X_{n+k, k}$ can be 2-regular if $k \geqq 3$, or $k$ $=2$ and $2 \nmid n$.

Let $f_{n}: S^{2 n+3} \rightarrow B U(n+1)$ and $g_{n}: S^{4 n+7} \rightarrow B S p(n+1)$ be the classifying maps of sphere bundles $S^{2 n+1} \rightarrow W_{n+2,2} \rightarrow S^{2 n+3}$ and $S^{4 n+3} \rightarrow X_{n+2,2} \rightarrow S^{4 n+7}$ respectively. We begin with the following exceptional cases.

Proposition 2.2. $W_{2,2}=U(2)$ is homeomorphic to $S^{1} \times S^{3}$ and $W_{4,2}$ is homeomorphic to $S^{5} \times S^{7}$.

Proof. The first part is obvious. For the second part, consider the composite $S^{7} \stackrel{f_{2}}{\longrightarrow} B U(3) \rightarrow B S O(6)$. There is an isomorphism of Lie groups $\operatorname{Spin}(6) \cong \operatorname{SU}(4) . \quad$ Hence $\quad \pi_{7}(B S O(6)) \cong \pi_{7}(B \operatorname{Spin}(6)) \cong \pi_{7}(B S U(4)) \cong \pi_{6}(S U(4))$ $=0$. Thus the above composition is null homotopic and $S^{5} \rightarrow W_{4,2} \rightarrow S^{7}$ is a trivial bundle with structure group $\mathrm{SO}(6)$.

We therefore concentrate on $W_{n+2,2}$ for even $n \geqq 4$, and on $X_{n+2,2}$ for $n \equiv 6$ modulo 8 (Recall Theorem 1.6). For the rest of this section, we localize all spaces and groups at prime 2 .

Theorem 203. ([5]) i) $\pi_{2 n+3}(B U(n+1)) \cong \pi_{2 n+2}(U(n+1)) \cong \mathbb{Z}_{(2)} /(n+1)$ !, and $f_{n}$ generates $\pi_{2 n+3}(B U(n+1))$.

ii) $\pi_{4 n+7}(B S p(n+1)) \cong \pi_{4 n+6}(\operatorname{Sp}(n+1)) \cong \mathbb{Z}_{(2)} / 2(2 n+3)$ ! for even $n$, $\cong \mathbb{Z}_{(2)} /(2 n+3)$ ! for odd $n$, and $g_{n}$ generates $\pi_{4 n+7}(B S p(n+1))$.

We also denote by $f_{n}, g_{n}$ the generator of $\pi_{2 n+2}(U(n+1)), \pi_{4 n+6}(\operatorname{Sp}(n+1))$ which correspond to $f_{n}, g_{n}$ respectively by the natural isomorphisms. Consider the following commutative diagrams.

$$
\begin{aligned}
& \pi_{2 n+3}(S O / S O(2 n+1)) \stackrel{\bar{j}_{*}}{\longrightarrow} \pi_{2 n+3}(S O / S O(2 n+2)) \stackrel{\bar{r}_{*}}{\longleftarrow} \pi_{2 n+3}(U / U(n+1)) \\
& \pi_{2 n+2}(S O(2 n+1)) \stackrel{j_{*}}{\longrightarrow} \quad \pi_{2 n+2}(S O(2 n+2)) \stackrel{r_{*}}{\longleftarrow} \pi_{2 n+2}(U(n+1)) \\
& \pi_{4 n+7}(S O / S O(4 n+3)) \stackrel{\bar{j}_{*}}{\longrightarrow} \pi_{4 n+7}(S O / S O(4 n+4)) \stackrel{\bar{r}_{*}^{\prime}}{\longleftarrow} \pi_{4 n+7}(\operatorname{Sp} / \operatorname{Sp}(n+1)) \\
& \pi_{4 n+6}(S O(4 n+3)) \stackrel{j_{*}}{\longrightarrow} \\
& \pi_{4 n+6}(S O(4 n+4)) \stackrel{r_{*}^{\prime}}{\longleftarrow}
\end{aligned}
$$
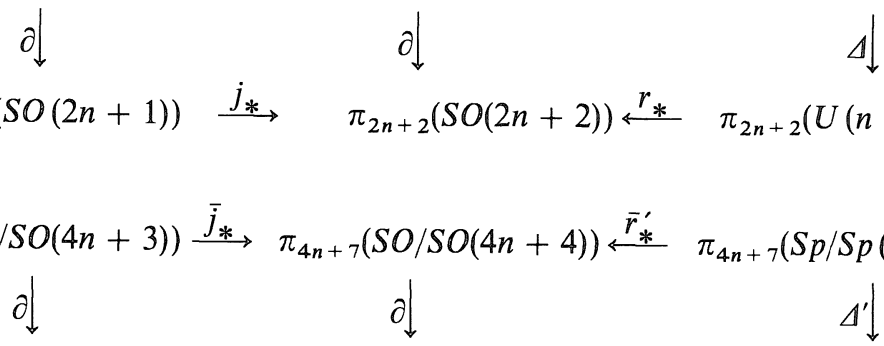
where $\partial, \Delta, \Delta^{\prime}$ are connecting homomorphisms, $r, r^{\prime}, j$ are inclusions $U(n$ $+1) \hookrightarrow S O(2 n+2), S p(n+1) \hookrightarrow S O(4 n+4), S O(m) \hookrightarrow S O(m+1)$, and $\bar{r}, \bar{r}^{\prime}, \bar{j}$ are the maps induced by $r, r^{\prime}, j$ respectively. Note that $\Delta, \Delta^{\prime}$ and both $\partial^{\prime}$ 's in (2.5) are surjective by the Bott periodicity. We choose elements $\bar{f}_{n} \in \pi_{2 n+3}(U / U(n$ $+1)$ ) and $\bar{g}_{n} \in \pi_{4 n+7}(S p / S p(n+1))$ such that $\Delta\left(\bar{f}_{n}\right)=f_{n}, \Delta^{\prime}\left(\bar{g}_{n}\right)=\bar{g}_{n}$.

Let $k: \mathbb{P}_{m}^{\infty} \rightarrow S O / S O(m)$ be the canonical inclusion. Since the pair $\left(S O / S O(m), \mathbf{P}_{m}^{\infty}\right)$ is $2 m$-connected, $k_{*}: \pi_{i}\left(\mathbf{P}_{m}^{\infty}\right) \rightarrow \pi_{i}(S O / S O(m))$ is an isomorphism for $i<2 m$.

Lemma 2.6. i) $k_{*}: \pi_{2 n+3}\left(\mathbb{P}_{2 n+i}^{\infty}\right) \rightarrow \pi_{2 n+3}(S O / S O(2 n+i))$ is an isomorphism for $i=1$, 2. If $n$ is even, we have $\pi_{2 n+3}\left(\mathbf{P}_{2 n+1}^{\infty}\right) \cong \mathbf{Z} / 8$ and $\pi_{2 n+3}\left(\mathbf{P}_{2 n+2}^{\infty}\right) \cong$ $\mathbb{Z} / 4$. Moreover, the mod 2 Hurewicz homomorphism $h: \pi_{2 n+3}\left(\mathbf{P}_{2 n+i}^{\infty}\right)$ $\rightarrow H_{2 n+3}\left(\mathbf{P}_{2 n+i}^{\infty} ; \mathbf{F}_{2}\right)(i=1,2)$ maps an element $x$ to a non-zero element if and only if $x$ generates $\pi_{2 n+3}\left(\mathbb{P}_{2 n+i}^{\infty}\right)$.

ii) $k_{*}: \pi_{4 n+7}\left(\mathbf{P}_{4 n+i}^{\infty}\right) \rightarrow \pi_{4 n+7}(S O / S O(4 n+i))$ is an isomorphism for $i=3$, 4 . If $n$ is even, we have $\pi_{4 n+7}\left(\mathbf{P}_{4 n+3}^{\infty}\right) \cong \mathbb{Z} / 16$ and $\pi_{4 n+7}\left(\mathbf{P}_{4 n+4}^{\infty}\right) \cong \mathbb{Z} / 16 \oplus \mathbb{Z} / 4$. Moreover, the mod 2 Hurewicz homomorphism $h: \pi_{4 n+7}\left(\mathbb{P}_{4 n+i}^{\infty}\right) \rightarrow H_{4 n+7}\left(\mathbb{P}_{4 n+i}^{\infty} ;\right.$ $\left.\mathbb{F}_{2}\right)(i=3,4)$ maps an element $x$ to a non-zero element if and only if the order of $x$ is 16 .

Proof. Consider the Adams spectral sequence $E_{2}^{s, t}=\operatorname{Ext}_{A_{*}}^{s, t}\left(\mathbb{F}_{2}, H_{*}\left(\mathbb{P}_{m}^{\infty}\right)\right)$ $\Rightarrow \pi_{t-s}^{S}\left(\mathbf{P}_{m}^{\infty}\right)$, where $A_{*}=\mathbb{F}_{2}\left[\xi_{1}, \xi_{2}, \ldots, \xi_{i}, \ldots\right]$ is the dual of the mod 2 Steenrod algebra. The $E_{2}$-terms we need are given as follows (See [10] Chapter III, and $[11])$;

$$
\begin{aligned}
& m=2 n+1: E_{2}^{s, 2 n+3+s}=\mathbb{F}_{2}\left\{h_{0}^{s} x_{2 n+3}\right\} \quad \text { for } s=0,1,2, \\
& E_{2}^{s, 2 n+3+s}=0 \text { for } s \geqq 3 \\
& m=2 n+2: E_{2}^{s, 2 n+3+s}=\mathbb{F}_{2}\left\{h_{0}^{s} x_{2 n+3}\right\} \text { for } s=0,1 \text {, } \\
& E_{2}^{s, 2 n+3+s}=0 \text { for } s \geqq 2 \\
& m=4 n+3: E_{2}^{s, 4 n+7+s}=\mathbb{F}_{2}\left\{h_{0}^{s} x_{4 n+7}\right\} \text { for } s=0,1,2,3 \text {, } \\
& E_{2}^{s, 4 n+7+s}=0 \text { for } s \geqq 4 \\
& m=4 n+4: E_{2}^{s, 4 n+7+s}=\mathbb{F}_{2}\left\{h_{0}^{s} x_{4 n+7}\right\} \text { for } s=0,3 \text {, } \\
& E_{2}^{s, 4 n+7+s}=\mathbb{F}_{2}\left\{h_{0}^{s} x_{4 n+7}, h_{0}^{s-1} h_{2} x_{4 n+4}\right\} \text { for } s=1,2, \\
& E_{2}^{s, 4 n+7+s}=0 \text { for } s \geqq 4,
\end{aligned}
$$

where $h_{i} \in \operatorname{Ext}_{A}^{1,2^{i}}\left(\mathbf{F}_{2}, \mathbf{F}_{2}\right)$ is the element represented by $\left[\xi_{1}^{2 i}\right]$ in the cobar complex of $A_{*}([1])$. It is also easy to see that $x_{2 n+3} \in E_{2}^{0,2 n+3}$ and $x_{4 n+7} \in E_{2}^{0,4 n+3}$ are permanent cycles in each spectral sequence and that $h_{1}^{s} x_{2 n+3}$ and $h_{1}^{s} x_{4 n+7}$ are not zero in the $E_{\infty}$-terms if they are not zero in the $E_{2}$-terms. Noting that $\pi_{2 n+3}\left(\mathbb{P}_{2 n+i}^{\infty}\right) \cong \pi_{2 n+3}^{S}\left(\mathbb{P}_{2 n+i}^{\infty}\right)(i=1,2)$ and $\pi_{4 n+7}\left(\mathbf{P}_{4 n+i}^{\infty}\right) \cong \pi_{4 n+7}^{S}\left(\mathbf{P}_{4 n+i}^{\infty}\right)(i=3,4)$, the result follows. 
Lemma 2.8. i) In diagram (2.4), $\bar{r}_{*}$ and $\bar{j}_{*}$ are surjective. And $\bar{r}_{*}\left(\bar{f}_{n}\right)$ generates $\pi_{2 n+3}(S O / S O(2 n+2))$.

ii) In diagram (2.5), $\bar{r}_{*}^{\prime}\left(\bar{g}_{n}\right)$ generates the images of $\bar{j}_{*}$ and $\bar{r}_{*}^{\prime}$ if $n \equiv 6$ modulo 8. And $\bar{j}_{*}$ is a split monomorphism.

Proof. We note that $\bar{r}_{*}: H_{2 n+3}\left(U / U(n+1) ; \mathbb{F}_{2}\right) \rightarrow H_{2 n+3}\left(S O / S O(2 n+2) ; \mathbb{F}_{2}\right)$ and $\bar{r}_{*}^{\prime}: H_{4 n+7}\left(S p / S p(n+1) ; \mathbb{F}_{2}\right) \rightarrow H_{4 n+7}\left(S O / S O(4 n+4) ; F_{2}\right)$ are isomorphisms, and that the mod 2 Hurewicz homomorphisms $h: \pi_{2 n+3}(U / U(n+1))$ $\rightarrow H_{2 n+3}\left(U / U(n+1) ; \mathbb{F}_{2}\right)$ and $h: \pi_{4 n+7}(\operatorname{Sp} / \operatorname{Sp}(n+1)) \rightarrow H_{4 n+7}\left(\operatorname{Sp} / \operatorname{Sp}(n+1) ; \mathbb{F}_{2}\right)$ map $\bar{f}_{n}, \bar{g}_{n}$ to each generator. It follows from the preceding lemma that $\bar{r}_{*}\left(\bar{f}_{n}\right)$ is a generator of $\pi_{2 n+3}(S O / S O(2 n+2))$ and that $\bar{r}_{*}^{\prime}\left(\bar{g}_{n}\right)$ is an element of order 16 . $r^{\prime}$ induces a map from a sphere bundle $S^{4 n+3} \rightarrow S p / S p(n) \rightarrow S p / S p(n+1)$ to a sphere bundle $S^{4 n+3} \rightarrow S O / S O(4 n+3) \rightarrow S O / S O(4 n+4)$. Then we have the following commutative diagram:

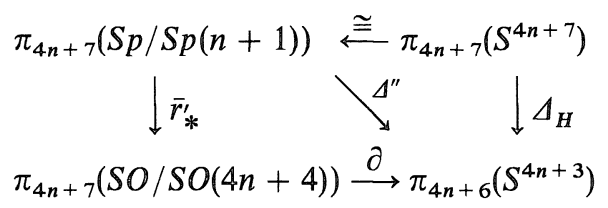

By Theorem 1.4, $\Delta_{H}=0$ if $n \equiv 6$ modulo 8 , hence $\partial \circ \bar{r}_{*}^{\prime}=\Delta^{\prime \prime}=0$ and the image of $\bar{r}_{*}^{\prime}$ is contained in the image of $\bar{j}_{*}$. The preceding lemma implies that $\bar{j}_{*}$ in (2.4) is surjective and that $\bar{j}_{*}$ in (2.5) is a split monomorphism. This completes the proof.

We put $H(n)=\left\{f: S^{n-1} \rightarrow S^{n-1} \mid f:\right.$ homotopy equivalence $\}$ and $S H(n)=\{f:$ $S^{n-1} \rightarrow S^{n-1} \mid$ degree $\left.f=1\right\}$ and give them the compact-open topology. We denote by $J_{S H}: S O(n) \rightarrow S H(n)$ and $j^{\prime}: S H(n) \rightarrow H(n)$ the canonical inclusions, and put $J_{H}=j^{\prime} \circ J_{S H}: S O(n) \rightarrow H(n) . H(n)$ and $S H(n)$ are topological monoids with respect to compositions of maps, and $J_{S H}, j^{\prime}$ and $J_{H}$ are maps of topological monoids. Let $e: S H(n) \rightarrow S^{n-1}$ be the evaluation map at the base point. We denote by $\Omega_{1}^{n-1} S^{n-1}$ the component of $\Omega^{n-1} S^{n-1}$ which contains the identity map. Then $\Omega_{1}^{n-1} S^{n-1}$ is contained in $S H(n)$ and we have a fibration $\Omega_{1}^{n-1} S^{n-1}$

$\rightarrow S H(n) \stackrel{e}{\longrightarrow} S^{n-1} . \quad J_{S H}$ induces $J: S O(n-1) \rightarrow \Omega_{1}^{n-1} S^{n-1}$ and $J_{*}: \pi_{i}(S O(n-1))$

$\rightarrow \pi_{i}\left(\Omega_{1}^{n-1} S^{n-1}\right) \cong \pi_{i+n-1}\left(S^{n-1}\right)$ coincides with the $J$-homomorphism ([23], [21] Chapter XI) up to sign. So, we rather denote $J_{*}$ by $J$.

By the construction of Dold-Lashof, $H(n)$ and $S H(n)$ have classifying spaces $B H(n)$ and $B S H(n)$. The following is a special case of Theorem 7.5 of [3].

Theorem 2.9. Let $S^{n-1} \rightarrow E \rightarrow B$ be an (n-1)-sphere bundle with structure group $S O(n)$ and let $f: B \rightarrow B S O(n)$ be the classifying map. Then $S^{n-1} \rightarrow E \rightarrow B$ is fiber homotopy equivalent to a trivial bundle if and only if the composition $B J_{H}^{\circ} \circ$ : $B \rightarrow B S O(n) \rightarrow B H(n)$ is null homotopic. 
We also need the following fact.

Proposition 2.10 ([8], Corollary 4.5). The sphere bundle $S^{a(n+1)-1} \rightarrow O_{n+k, k}$ $\rightarrow O_{n+k, k-1}$ is fiber homotopy equivalent to a trivial bundle if and only if $O_{n+k, k}$ is homotopy equvalent to $S^{d(n+1)-1} \times O_{n+k, k-1}$, where $O_{n+k, k}=V_{n+k, k}, W_{n+k, k}$ or $X_{n+k, k}$ and $d=1,2$ or 4 , respectively.

Now we reduce the problem to a problem on the $J$-homomorphism.

Lemma 2.11. i) For even $n, W_{n+2,2}$ is not 2-regular if the image of $J$ : $\left.\pi_{2 n+2}(S O(2 n+1)) \rightarrow \pi_{2 n+2}\left(\Omega_{1}^{2 n+1} S^{2 n+1}\right)\right)$ contains an element of order four.

ii) For $n \equiv 6$ modulo $8, X_{n+2,2}$ is not 2-regular if $J: \pi_{4 n+6}(\operatorname{SO}(4 n+3))$ $\rightarrow \pi_{4 n+6}\left(\Omega_{1}^{4 n+3} S^{4 n+3}\right)$ is non-trivial.

Proof. By the exactness of $\pi_{2 n+3}\left(S^{2 n+1}\right) \rightarrow \pi_{2 n+2}\left(\Omega_{1}^{2 n+1} S^{2 n+1}\right) \stackrel{j_{*}^{\prime \prime}}{\rightarrow} \pi_{2 n+2}$ $(S H(2 n+2))$, the order of the kernel of $j_{*}^{\prime \prime}: \pi_{2 n+2}\left(\Omega_{1}^{2 n+1} S^{2 n+1}\right) \rightarrow \pi_{2 n+2}(S H(2 n$ $+2)$ ) is at most two. Hence the assumption of i) implies that $J_{S_{H^{*}}}: \pi_{2 n+2}(\mathrm{SO}(2 n$ $+2)) \rightarrow \pi_{2 n+2}(S H(2 n+2))$ is non-trivial. Since $f_{n}$ generates $\pi_{2 n+2}(U(n+1))$ and $r_{*}: \pi_{2 n+2}(U(n+1)) \rightarrow \pi_{2 n+2}(S O(2 n+2))$ is surjective by Lemma 2.8 , it follows that $J_{H} \circ{ }^{\circ} \circ f_{n}$ is non-trivial in $\pi_{2 n+2}(H(2 n+2))$. Therefore $B J_{H} \circ B r \circ f_{n}$ is nontrivial in $\pi_{2 n+3}(B H(2 n+2))$, and the result follows from Theorem 2.9 and Proposition 2.10. For the part ii), note that $j_{*}^{\prime \prime}: \pi_{4 n+6}\left(\Omega_{1}^{4 n+3} S^{4 n+3}\right) \rightarrow$ $\pi_{4 n+6}(S H(4 n+4))$ is injective since $\pi_{4 n+7}\left(S^{4 n+3}\right)=0$. Then, the assumption of ii) implies the non-triviality of $J_{S H} \circ r^{\prime} \circ g_{n}$. In fact, by the commutativity of (2.5) and Lemma 2.8, there exists a generator $\gamma_{n}$ of $\pi_{4 n+7}(S O / S O(4 n+3))$ such that $\bar{r}_{*}^{\prime}\left(\bar{g}_{n}\right)=\bar{j}_{*}\left(\gamma_{n}\right)$ and we have $J_{S H^{*}} \circ r_{*}^{\prime}\left(g_{n}\right)=J_{S H^{*}} \circ \partial \circ \partial \circ \bar{r}_{*}^{\prime}\left(\bar{g}_{n}\right)=J_{S H^{*}} \circ j_{*} \circ \partial\left(\gamma_{n}\right)$ $=j_{*}^{\prime \prime} \circ J \circ \partial\left(\gamma_{n}\right) \neq 0$ since $\partial$ is surjective and $j_{*}^{\prime \prime} \circ J \neq 0$. Therefore $B J_{H} \circ B r^{\prime} \circ g_{n} \neq 0$ in $\pi_{4 n+7}(B H(4 n+4))$.

In order to examine the $J$-homomorphisms, we use the relative $J$ homomorphisms constructed by Toda ([21]). We briefly review the construction.

For a pair $(X, A)$ of topological spaces with base point $* \in A$, we denote by $\Omega(X, A)$ a topological space $\{l:[0,1] \rightarrow X \mid l$ : continuous, $l(0) \in A, l(1)=*\}$ with compact-open topology. Let $e: \Omega(X, A) \rightarrow A$ be the evaluation map at 0 . Then $\Omega(X, A)$ is the homotopy fiber of inclusion map $A \rightarrow X$ and $\Omega X \stackrel{\delta}{\longrightarrow} \Omega(X, A) \stackrel{e}{\longrightarrow} A \rightarrow X$ is a fiber sequence, where $\delta$ is the natural inclusion.

The natural inclusion $i: X \rightarrow \Omega \Sigma X$ is extended to a map $i^{\prime}: X \cup C A \rightarrow \Omega(\Sigma X$, $\Sigma A)$ defined by $i^{\prime}([a, t])(s)=[a, s t+1-t]$ on $C A$, where $X \cup C A=(X \amalg(A$ $\times I)) / \sim$, and $\sim$ is an equivalence relation generated by $a \sim(a, 1),(a, 0) \sim *$ $\sim(*, t)$ for $a \in A, t \in[0,1]$. We denote by $[a, t]$ the element of $X \cup C A$ (or $\Sigma A$ ) represented by $(a, t) \in A \times I$. 
The map $J: S O(m) \rightarrow \Omega_{1}^{m} S^{m}$ induces the stable $J$-map $J^{S}: S O \rightarrow Q_{1} S^{0}$, and let $J^{\prime}: \Sigma^{m} S O(m) \rightarrow S^{m}$ and $J_{m}^{S}: \Sigma^{m} S O \rightarrow Q S^{m}$ be the adjoint maps of $J$ and $J^{s}$. We put $\quad F(m)=\Omega\left(Q S^{m}, S^{m}\right)$, then $J^{\prime}$ and $J_{m}^{S}$ induce $J^{R}: \Omega\left(\Sigma^{m} S O, \Sigma^{m} S O(m)\right)$ $\rightarrow F(m)$. We define the relative $J$-map $J^{r e l}: \Sigma^{m-1} \mathbb{P}_{m}^{\infty} \rightarrow F(m)$ to be the composition

$\Sigma^{m-1} \mathbb{P}_{m}^{\infty} \stackrel{i n v}{\longrightarrow} \Sigma^{m-1}\left(\mathbb{P}^{\infty} \cup C \mathbb{P}^{m-1}\right) \stackrel{i^{\prime}}{\longrightarrow} \Omega\left(\Sigma^{m} \mathbb{P}^{\infty}, \Sigma^{m} \mathbb{P}^{m-1}\right) \stackrel{k^{\prime}}{\longrightarrow} \Omega\left(\Sigma^{m} S O, \Sigma^{m} S O(m)\right)$

$\stackrel{J^{R}}{\longrightarrow} F(m)$,

where inv is the $(m-1)$-fold suspension of the homotopy inverse of the collapsing map $\mathbb{P}^{\infty} \cup C \mathbb{P}^{m-1} \rightarrow \mathbb{P}_{m}^{\infty}\left(\mathbb{P}^{n}=\mathbb{R}^{n}\right)$, and $k^{\prime}$ is induced by the canonical inclusion $\mathbb{P}^{\infty} \subseteq S O$.

Let $\rho: S O \rightarrow S O / S O(m)$ be the projection, then $\left(\Sigma^{m} \rho\right)_{*}: \pi_{j}\left(\Sigma^{m} S O, \Sigma^{m} S O(m)\right)$ $\rightarrow \pi_{j}\left(\Sigma^{m}(S O / S O(m))\right)$ is an isomorphism for $j<3 m$. In fact, let $Y$ be the homotopy fiber of $\Sigma^{m} \rho$, then there is a map $\zeta: \Sigma^{m} S O(m) \rightarrow Y$ such that the composition $\Sigma^{m} S O(m) \stackrel{\zeta}{\longrightarrow} Y \rightarrow \Sigma^{m} S O$ is homotopic to inclusion $\Sigma^{m} S O(m) \hookrightarrow \Sigma^{m} S O$.

By an easy argument on the Serre spectral sequence associated with fibering $Y$ $\rightarrow \Sigma^{m} S O \rightarrow \Sigma^{m}(S O / S O(m))$, we see that $\zeta_{*}: H_{j}\left(\Sigma^{m} S O(m)\right) \rightarrow H_{j}(Y)$ is an isomorphism for $j<3 m$ and an epimorphism for $j \leqq 3 m$. Thus $\zeta_{*}: \pi_{j}\left(\Sigma^{m} S O(m)\right) \rightarrow \pi_{j}(Y)$ is an isomorphism for $j<3 m$ and the above assertion follows from the fivelemma.

We denote by $\bar{k}: \mathbb{P}^{\infty} \cup C \mathbb{P}^{m-1} \rightarrow S O / S O(m)$ the composition $\mathbb{P}^{\infty} \cup C \mathbb{P}^{m-1}$ $\rightarrow \mathbb{P}_{m}^{\infty} \stackrel{k}{\longrightarrow} S O / S O(m)$. For $j<2 m-1$, we have the following commutative diagram

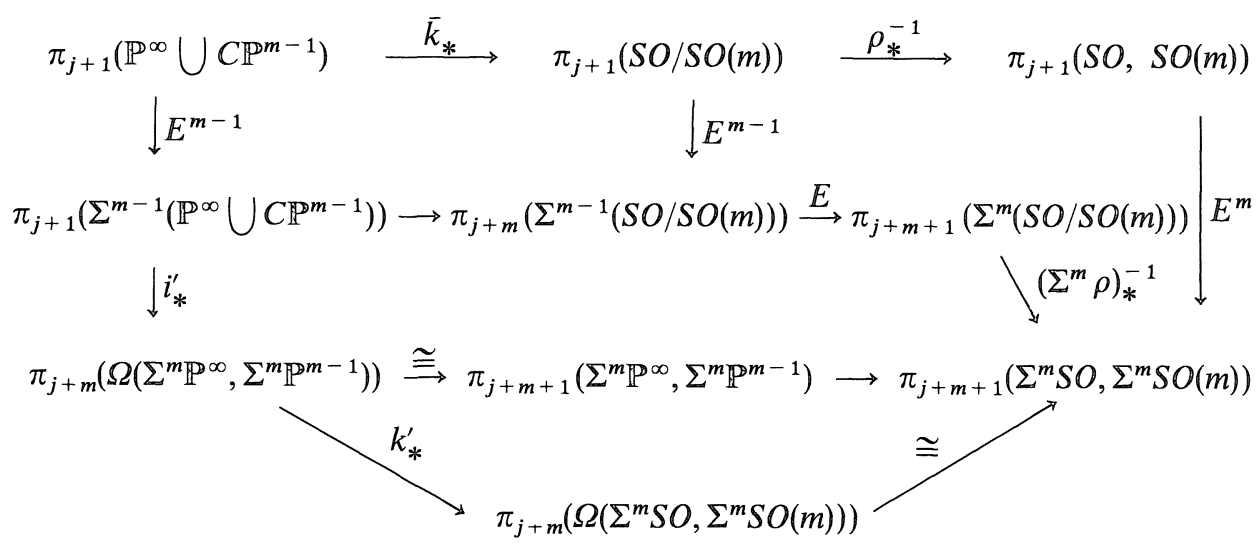

By the definition of the relative $J$-map and the commutativity of the above diagram, the following diagram commutes for $j<2 m-1$. 


$$
\begin{gathered}
\pi_{j+1}\left(\mathbb{P}_{m}^{\infty}\right) \stackrel{k_{*}}{\longrightarrow} \pi_{j+1}(S O / S O(m)) \stackrel{\partial}{\longrightarrow} \pi_{j}(S O(m)) \\
\downarrow E^{m-1} \\
\pi_{j+m}\left(\Sigma^{m-1} \mathbb{P}_{m}^{\infty}\right) \stackrel{J_{*}^{r e l}}{\longrightarrow} \pi_{j+m}(F(m)) \stackrel{e_{*}}{\longrightarrow} \pi_{j+m}\left(S^{m}\right) \cong \pi_{j}\left(\Omega_{1}^{m} S^{m}\right)
\end{gathered}
$$

Theorem $2.13\left([21]\right.$, Theorem 11.7). $\quad J_{*}^{r e l}: \pi_{j}\left(\Sigma^{m-1} \mathbf{P}_{m}^{\infty}\right) \rightarrow \pi_{j}(F(m))$ is an isomorphism for $j<4 m-3$.

In the case $m=2 n+1, j=2 n+2$ ( $n$ : even) or $m=4 n+3, j=4 n+6$ ( $n \equiv 6$ modulo 8 ), $k_{*}, E^{m-1}$ and $J_{*}^{r e l}$ are isomorphisms and $\partial$ is an epimorphism in the diagram (2.12). Consider the exact sequence associated with fiber sequence $Q S^{m-1} \stackrel{\delta}{\longrightarrow} F(m) \stackrel{e}{\longrightarrow} S^{m}$. Then the following lemma is obvious since $\pi_{4 n+3}(F(2 n+1)) \cong \mathbb{Z} / 8$ if $n$ is even by Lemma 2.8 and the above theorem. $\left(\pi_{8 n+9}(F(4 n+3))\right.$ is a cyclic group of order sixteen.)

Lemma 2.14. i) For even $n$, the image of $J: \pi_{2 n+2}(S O(2 n+1)) \rightarrow$ $\pi_{2 n+2}\left(\Omega_{1}^{2 n+1} S^{2 n+1}\right)$ contains an element of order four, if the order of the image of $\delta_{*}: \pi_{4 n+3}\left(Q S^{2 n}\right) \rightarrow \pi_{4 n+3}(F(2 n+1))$ is at most two.

ii) For $n \equiv 6$ modulo $8, J: \pi_{4 n+6}(S O(4 n+3)) \rightarrow \pi_{4 n+6}\left(\Omega_{1}^{4 n+3} S^{4 n+3}\right)$ is non-trivial if $\delta_{*}: \pi_{8 n+9}\left(Q S^{4 n+2}\right) \rightarrow \pi_{8 n+9}(F(4 n+3))$ is not surjective.

Thus it suffices to examine $\delta_{*}: \pi_{j}\left(Q S^{m-1}\right) \rightarrow \pi_{j}(F(m))$ in the above cases. We apply the following theorem which was first given by Mahowald ([10], Theorem A), and later it was reformulated by Milgram ([14], Part II) using a kind of unstable Adams spectral sequence. For a space $X$, we denote by $\left\{E_{r}^{s, t}(X), d_{r}\right\}$ a certain unstable Adams spectral sequence constructed in [14], Part II. It converges to $\pi_{*}(X) \otimes \mathbb{Z}_{2}^{\wedge}$ for "good" $X(X=\Sigma Y$, for example), and in the stable range, it coincides with the stable Adams spectral sequence. Thus $E_{2}^{s, t}(X) \cong \operatorname{Ext}_{A_{*}}^{s, t}\left(\mathbb{F}_{2}, \widetilde{H}_{*}(X)\right)$ for $t-s<2 n-1$ if $X$ is $(n-1)$-connected. In fact, there is a map of spectral sequences $E_{r}^{s, t}(X) \rightarrow E_{r}^{s, t+1}(\Sigma X)$ and let $\left\{E_{r}^{s, t}\left(\Sigma^{\infty} X\right), d_{r}\right\}$ be the spectral sequence defined by $E_{r}^{s, t}\left(\Sigma^{\infty} X\right)=\underline{\lim _{u}} E_{r}^{s, t+u}\left(\Sigma^{u} X\right)$, then $\left\{E_{r}^{s, t}\left(\Sigma^{\infty} X\right), d_{r}\right\}$ is the stable Adams spectral sequence (See [14], §6 for details). Note that $F(m)$ is $(2 m-2)$-connected and it is homotopy equivalent to $\Sigma^{m-1} \mathbb{P}^{\infty}$ through dimension $4 m-4$ by Theorem 2.13. Hence $\left\{E_{r}^{s, t}(F(m)), d_{r}\right\}$ converges to $\pi_{t-s}(F(m)) \otimes \mathbb{Z}_{2}^{\hat{2}} \quad$ and $\quad E_{2}^{s, t}(F(m)) \cong \operatorname{Ext}_{A_{*}}^{s, t}\left(\mathbb{F}_{2}, \quad \widetilde{H}_{*}(F(m))\right) \cong$ $\operatorname{Ext}_{A_{*}}^{s, t-m+1}\left(\mathbb{F}_{2}, \widetilde{H}_{*}\left(\mathbb{P}_{m}^{\infty}\right)\right)$ for $t-s<4 m-3$.

Theorem 2.15 ([10] Theorem A, [14] Theorem 8.5 and Theorem 8.21).

There is a map of spectral sequences $\delta_{r}: E_{r}^{s, t}\left(\Sigma^{\infty} S^{m-1}\right) \rightarrow E_{r}^{s-1, t-1}(F(m))$ which converges to $\delta_{*}: \pi_{t-s}^{S}\left(S^{m-1}\right) \cong \pi_{t-s}\left(Q S^{m-1}\right) \rightarrow \pi_{t-s}(F(m))$ for $t-s<3 m-1$. In the $E_{2}$-term, $\delta_{2}$ is identified with a homomorphism $\operatorname{Ext}_{A_{*}}^{s, t}\left(\mathbb{F}_{2}, \widetilde{H}_{*}\left(S^{m-1}\right)\right)$ 
$\rightarrow \operatorname{Ext}_{A_{*}}^{s-1, t-1}\left(\mathbb{F}_{2}, \widetilde{H}_{*}\left(\Sigma^{m-1} \mathbb{P}_{m}^{\infty}\right)\right)$ for $t-s<3 m-1$.

Lemma 2.16 i) If $n$ is even and greater than 2, the order of the image of $\delta_{*}$ : $\pi_{4 n+3}\left(Q S^{2 n}\right) \rightarrow \pi_{4 n+3}(F(2 n+1))$ is at most 2 .

ii) If $n$ is even and positive, $\delta_{*}: \pi_{8 n+9}\left(Q S^{4 n+2}\right) \rightarrow \pi_{8 n+9}(F(4 n+3))$ is not surjective.

Proof. We denote by $F^{s, t}\left(\Sigma^{\infty} S^{m-1}\right), F^{s, t}(F(m))$ the filtrations on $\pi_{*}\left(Q S^{m-1}\right)$ and $\pi_{*}(F(m))$ associated with the corresponding spectral sequences in Theorem 2.15. Since $E_{\infty}^{s, s+j}\left(\Sigma^{\infty} S^{m-1}\right)=0$ for $s=0,1,2$ if $j$ and $m$ are odd and $j \neq m, m$ $+2, m+6$ ([1] for $s=1,[11]$ for $s=2)$, it follows that $\pi_{j}\left(Q S^{m-1}\right)=\pi_{j}^{S}\left(S^{m-1}\right)$ $=F^{3, j+3}\left(\Sigma^{\infty} S^{m-1}\right)$. Hence, by Theorem 2.15, we see that $\delta_{*}\left(\pi_{4 n+3}\left(Q S^{2 n}\right)\right)$ $\subset F^{2,4 n+5}(F(2 n+1))$ if $n>2$ and that $\delta_{*}\left(\pi_{8 n+9}\left(Q S^{4 n+2}\right)\right) \subset F^{2,8 n+11}(F(4 n+3))$ if $n>0$. On the other hand, since $E_{2}^{s, t}(F(m)) \cong \operatorname{Ext}_{A_{*}}^{\mathrm{s}, t-m+1}\left(\mathbb{F}_{2}, \tilde{H}_{*}\left(\mathbb{P}_{m}^{\infty}\right)\right)$, it follows from (2.7) that $F^{2,4 n+5}(F(2 n+1)) \cong \mathbb{Z} / 2$ and $F^{2,8 n+11}(F(4 n+3)) \cong \mathbb{Z} / 4$ if $n$ is even. This completes the proof.

Thus, by Lemmas $2.11,2.14$ and 2.16 , we have proved

Theorem 2.17. $W_{n+2,2}$ is not 2-regular unless $n=0$ or 2 , and $X_{n+2,2}$ is not 2-regular for any $n \geqq 0$.

Let $\phi: S^{4 n+3} \rightarrow Q_{n+2,2}(\mathbb{C})$ be the attaching map of the top cell of $W_{n+2,2}$. If $n$ is even, we have a collapsing map $c: Q_{n+2,2}(\mathbb{C}) \cong S^{2 n+1} \vee S^{2 n+3} \rightarrow S^{2 n+1}$ by Theorem 1.3. Proposition 2.10 and Theorem 2.17 imply that the composition $S^{4 n+3} \stackrel{\phi}{\longrightarrow} Q_{n+2,2}(\mathbb{C}) \rightarrow S^{2 n+1}$ is not null homotopic if $n \geqq 4$. Otherwise, we can extend $c$ to $\bar{c}: W_{n+2,2} \rightarrow S^{2 n+1}$ which is a left homotopy inverse of the canonical inclusion $S^{2 n+1} \rightarrow W_{n+2,2}$. Then, fibration $S^{2 n+1} \rightarrow W_{n+2,2} \rightarrow S^{2 n+3}$ becomes retractible in the sense of James $([8])$, it is fiber homotopy equivalent to the trivial bundle ([8], Corollary 4.3). This contradicts Theorem 2.17. Hence we have a family of non-trivial elements $\phi_{j}$ in the 2-component of $\pi_{8 j+3}\left(S^{4 j+1}\right)(j$ $\geqq 2$ ). Similarly, the attaching maps of the top cell of $X_{n+2,2}$ define a family of non-trivial elements $\psi_{j}$ in the 2 -component of $\pi_{64 j+57}\left(S^{32 j+27}\right)(j \geqq 0)$.

Corollary 2.18. The attaching maps of the top cells of $W_{n+2,2}$ and $X_{n+2,2}$ define non-trivial elements $\phi_{j}(j \geqq 2)$ and $\psi_{j}(j \geqq 0)$ in the 2-components of $\pi_{8 j+3}\left(S^{4 j+1}\right)$ and $\pi_{64 j+57}\left(S^{32 j+27}\right)$. Moreover, $\phi_{j}$ is in the kernel of $E^{3}$ : $\pi_{8 j+3}\left(S^{4 j+1}\right) \rightarrow \pi_{8 j+6}\left(S^{4 j+4}\right) \cong \pi_{4 j+2}^{S}\left(S^{0}\right)$ and has order at most eight, $\psi_{j}$ is in the kernel of $E^{5}: \pi_{64 j+57}\left(S^{32 j+27}\right) \rightarrow \pi_{64 j+62}\left(S^{32 j+32}\right) \cong \pi_{32 j+30}^{S}\left(S^{0}\right)$ and has order at most sixteen.

Proof. The assertion that $\phi_{j} \in \operatorname{Ker} E^{3}$ and $\psi_{j} \in \operatorname{Ker} E^{5}$ is shown in Appendix (See the proof of Corollary A.10.). By the exactness of $\pi_{*}(F(m))$ $\stackrel{e_{*}}{\rightarrow} \pi_{*}\left(S^{m}\right) \stackrel{i_{*}}{\rightarrow} \pi_{*}\left(Q S^{m}\right), \quad \phi_{j} \in \operatorname{Ker} E^{3}$ and $\quad \psi_{j} \in \operatorname{Ker} E^{5}$ imply that $\phi_{j} \in \operatorname{Im}\left[e_{*}\right.$ : 
$\left.\pi_{8 j+3}(F(4 j+1)) \rightarrow \pi_{8 j+3}\left(S^{4 j+1}\right)\right]$ and $\psi_{j} \in \operatorname{Im}\left[e_{*}: \pi_{64 j+57}(F(32 j+27)) \rightarrow \pi_{64 j+57}\right.$ $\left.\left(S^{32 j+27}\right)\right]$. Then the assertion $8 \phi_{j}=16 \psi_{j}=0$ follows from $\pi_{8 j+3}(F(4 j+1)) \cong$ $\pi_{8 j+3}\left(\Sigma^{4 j} \mathbb{P}_{4 j+1}^{\infty}\right)=\mathbb{Z} / 8$ and $\pi_{64 j+57}(F(32 j+27)) \cong \pi_{64 j+57}\left(\Sigma^{32 j+26} \mathbb{P}_{32 j+27}^{\infty}\right)$ $\cong \mathbb{Z} / 16$.

Remark 2.19. If $n$ is even and greater than two, Theorem 2.17 implies that spaces $\Omega W_{n+2,2}$ and $\Omega\left(S^{2 n+1} \times S^{2 n+3}\right)$ are different as loop spaces (with the natural loop space structures), although they have same homotopy type by Proposition 1.10. Therefore both $\Omega W_{n+2,2}$ and $\Omega\left(S^{2 n+1} \times S^{2 n+3}\right)$ have at least two different loop space structures. One is the natural one and the other is induced by the homotopy equivalence $\Omega W_{n+2,2} \simeq \Omega\left(S^{2 n+1} \times S^{2 n+3}\right)$ in Proposition 1.10. Hence, if we deloop $\Omega W_{n+2,2}$ and $\Omega\left(S^{2 n+1} \times S^{2 n+3}\right)$ with respect to the exotic loop space structures, we have homotopy equivalences $B \Omega W_{n+2,2}$ $\simeq S^{2 n+1} \times S^{2 n+3}$ and $B \Omega\left(S^{2 n+1} \times S^{2 n+3}\right) \simeq W_{n+2,2}$. Similarly, if $n \equiv 6$ modulo $8, \Omega X_{n+2,2}$ and $\Omega\left(S^{4 n+3} \times S^{4 n+7}\right)$ have exotic loop space structures.

We also remark that $\Sigma^{3} W_{n+2,2}$ (resp. $\Sigma^{5} X_{n+2,2}$ ) is homotopy equivalent to $\Sigma^{3}\left(S^{2 n+1} \times S^{2 n+3}\right)\left(\right.$ resp. $\left.\Sigma^{5}\left(S^{4 n+3} \times S^{4 n+7}\right)\right)$ for $n$ as above since the attaching of the top cell suspends to zero in the stable range. Details are left to readers.

\section{$\S 3$. On the Homotopy Fiber of $S^{2 n+1} \rightarrow Q S^{2 n+1}$}

Throughout this section, $p$ is a fixed odd prime and all spaces, spectra and groups are localized at prime $p$. As in the previous section, we denote by $F(n)$ the homotopy fiber of the canonical inclusion $i: S^{n} \rightarrow Q S^{n}$. The structure of $H_{*}\left(Q S^{n} ; \mathbb{F}_{p}\right)$ is described in [4] and [12]. We review briefly according to [12].

For a sequence $I=\left(\varepsilon_{1}, s_{1}, \ldots, \varepsilon_{k}, s_{k}\right)$ of non-negative integers such that $\varepsilon_{j}=0$ or 1 and $s_{j} \geqq \varepsilon_{j}$, define the degree, length and excess of $I$ by $d(I)=\sum_{j=1}^{k}\left(2 s_{j}(p\right.$ $\left.-1)-\varepsilon_{j}\right), l(I)=k$ and $e(I)=2\left(p s_{1}-\varepsilon_{1}\right)-d(I)$ respectively. We denote by $Q^{I}$ the iterated homology operation $\beta^{\varepsilon_{1}} Q^{s_{1}} \cdots \beta^{\varepsilon_{k}} Q^{s_{k}}$. I is said to be admissible if $p s_{j}$ $-\varepsilon_{j} \geqq s_{j-1}$ for $2 \leqq j \leqq k$. We also call the empty sequence $I$ admissible and assign $d(I)=l(I)=0, e(I)=\infty, Q^{I}=$ (identity operation).

Theorem 3.1. ([4], [12]). For a positive integer $n$, let $\iota_{n} \in H_{n}\left(Q S^{n}\right)$ be the image of the canonical generator of $H_{n}\left(S^{n}\right)$ by $i_{*}: H_{n}\left(S^{n}\right) \rightarrow H_{n}\left(Q S^{n}\right)$. And let $A_{n}$ be the free commutative graded algebra over $\mathbb{F}_{p}$ generated by $T_{n}=\left\{Q^{I} l_{n} \mid I\right.$ : admissible, $e(I) \geqq n\}$, that is, $A_{n}$ is the tensor product of an exterior algebra $E\left(Q^{I} l_{n} \mid I:\right.$ admissible, $e(I) \geqq n, n+d(I)$ : odd $)$ and a polynomial algebra $\mathbb{F}_{p}\left[Q^{I} l_{n} \mid I\right.$ : admissible, $e(I) \geqq n, n+d(I)$ : even $]$. Let $B_{n}$ be an ideal of $A_{n}$ generated by $\left\{Q^{s} x\right.$ $\left.-x^{p} \mid x \in T_{n}, \operatorname{deg} x=2 s\right\}$. Then $H_{*}\left(Q S^{n}\right)$ is isomorphic to $A_{n} / B_{n}$ as an algebra and each generator $Q^{I} l_{n}$ is primitive.

Corollary 3.2. $i_{*}: \pi_{t}\left(S^{2 n+1}\right) \rightarrow \pi_{t}\left(Q S^{2 n+1}\right)$ is an isomorphism for $t<2 p n$ $+2 p-3$ and an epimorphism for $t<2 p n+2 p-2$. Hence $F(2 n+1)$ is $(2 p n$ $+2 p-4)$-connected. 
Proof. Since $H_{s}\left(Q S^{2 n+1}\right)=0$ for $2 n+1<s<2 p n+2 p-2, i_{*}: H_{t}\left(S^{2 n+1}\right)$ $\rightarrow H_{t}\left(Q S^{2 n+1}\right)$ is an isomorphism for $t<2 p n+2 p-2$.

Let us consider the following fibration in order to compute the mod $p$ homology of $F(2 n+1)$.

$$
Q S^{2 n} \stackrel{\delta}{\longrightarrow} F(2 n+1) \stackrel{e}{\longrightarrow} S^{2 n+1}
$$

Note that the above fibration is a principal fibration, hence by applying the Wang sequence we have

Lemma 3.4. $\delta_{*}: H_{*}\left(Q S^{2 n}\right) \rightarrow H_{*}(F(2 n+1))$ is surjective and its kernel is an ideal generated by $l_{2 n}$. Therefore $H_{*}(F(2 n+1)) \cong H_{*}\left(Q S^{2 n}\right) /\left(l_{2 n}\right)$.

Since $F(2 n+1)$ has homotopy type of a simply connected $C W$-complex, we may replace $F(2 n+1)$ by a homotopy equivalent normal $C W$-complex $\widetilde{F}(2 n$ $+1)$. Thus the inclusion map of the $\left(2 n p+2 p^{2}-4\right)$-skeleton of $\widetilde{F}(2 n+1)$ induces isomorphism of homology groups through dimension $2 n p+2 p^{2}-4$. Define a $(-1)$-connected suspension spectrum $L_{n}$ to be $\Sigma^{-2 n p-2 p+3} \tilde{F}(2 n$ $+1)^{2 n p+2 p^{2}-4}$. Let $y_{i}, z_{i} \in H_{*}\left(L_{n}\right)$ be the elements corresponding to $\delta_{*}\left(\beta Q^{n+i+1} l_{2 n}\right)$ and $\delta_{*}\left(Q^{n+i+1} l_{2 n}\right)$ in $H_{*}(F(2 n+1))$. Hence $\operatorname{deg} y_{i}=2 i(p-1)$, $\operatorname{deg} z_{i}=2 i(p-1)+1$ and $\left\{y_{0}, z_{0}, y_{1}, z_{1}, \ldots y_{p-1}, z_{p-1}\right\}$ is a basis of $H_{*}\left(L_{n}\right)$ if $n$ $\geqq p-1$. So, from now on, we assume $n \geqq p-1$ for simplicity unless otherwise stated. Let $A_{*}$ be the dual mod $p$ Steenrod algebra, then $A_{*}$ $=E\left(\tau_{0}, \tau_{1}, \ldots\right) \otimes \mathbb{F}_{p}\left[\xi_{1}, \xi_{2}, \ldots\right]$ as an algebra $([16])$.

Lemma 3.5. The coaction $\psi: H_{*}\left(L_{n}\right) \rightarrow A_{*} \otimes H_{*}\left(L_{n}\right)$ is given by

$$
\begin{aligned}
\psi\left(y_{i}\right)=\Sigma_{j=0}^{i}\left(\begin{array}{c}
n+i+1 \\
j
\end{array}\right) \xi_{1}^{i} \otimes y_{i-j} \\
\begin{aligned}
\psi\left(z_{i}\right)=\Sigma_{j=0}^{i} & \left(\begin{array}{c}
n+i \\
j
\end{array}\right) \xi_{1}^{j} \otimes z_{i-j}+\Sigma_{j=0}^{i}\left(\begin{array}{c}
n+i+1 \\
j
\end{array}\right) \tau_{0} \xi_{1}^{j} \otimes y_{i-j} \\
& -\Sigma_{j=1}^{i}\left(\begin{array}{c}
n+i \\
j-1
\end{array}\right) \tau_{1} \xi_{1}^{j-1} \otimes y_{i-j} .
\end{aligned}
\end{aligned}
$$

Proof. The Nishida relations ([17], see also [12]) determine the action of $P_{*}^{j}$ on $y_{i}, z_{i}$ as follows.

$$
\begin{aligned}
& P_{*}^{j} y_{i}=(-1)^{j}\left(\begin{array}{c}
(n+i-j+1)(p-1)-1 \\
j
\end{array}\right) y_{i-j}, \\
& P_{*}^{j} z_{i}=(-1)^{j}\left(\begin{array}{c}
(n+i-j+1)(p-1) \\
j
\end{array}\right) z_{i-j} \text { and } \beta z_{i}=y_{i}
\end{aligned}
$$

The result easily follows from the above and congruences 


$$
\begin{aligned}
& (-1)^{j}\left(\begin{array}{c}
(m-j)(p-1)-1 \\
j
\end{array}\right) \equiv\left(\begin{array}{c}
m \\
j
\end{array}\right) \\
& (-1)^{j}\left(\begin{array}{c}
(m-j)(p-1) \\
j
\end{array}\right) \equiv\left(\begin{array}{c}
m-1 \\
j
\end{array}\right) \text { modulo } p \text { for } 0 \leqq j \leqq p-1 .
\end{aligned}
$$

Let $P_{*}=\mathbb{F}_{p}\left[\xi_{1}, \xi_{2}, \ldots\right]$ be the Hopf subalgebra of $A_{*}$ generated by $\xi_{1}, \xi_{2}, \ldots$ and put $E_{*}=A_{*} / / P_{*}=E\left(\tau_{0}, \tau_{1}, \ldots\right)$. Then $P_{*} \rightarrow A_{*} \rightarrow E_{*}$ is an extension of Hopf algebras. To compute the $B P$-homology of $L_{n}$, we use the Adams spectral sequence $\quad E_{2}^{s, t}=\mathrm{Ext}_{A_{*}}^{s, t}\left(\mathbb{F}_{p}, H_{*}\left(B P \wedge L_{n}\right)\right) \Rightarrow B P_{t-s}\left(L_{n}\right)$. First note that $H_{*}\left(B P \wedge L_{n}\right) \cong P_{*} \otimes H_{*}\left(L_{n}\right)$ is isomorphic to $A_{*} \square_{E_{*}} H_{*}\left(L_{n}\right)$ as an $A_{*^{-}}$ comodule. In fact, define $f: P_{*} \otimes H_{*}\left(L_{n}\right) \rightarrow A_{*} \otimes H_{*}\left(L_{n}\right)$ by $f(\alpha \otimes x)$ $=\Sigma_{i} \alpha a_{i} \otimes x_{i}$ where $\psi(x)=\Sigma_{i} a_{i} \otimes x_{i}$, then $f$ lifts to $\bar{f}: P_{*} \otimes H_{*}\left(L_{n}\right)$ $\rightarrow A_{*} \square_{E_{*}} H_{*}\left(L_{n}\right)$ which is an isomorphism of $A_{*}$-comodules. We apply the Change-of-Rings isomorphism and have

$$
\begin{aligned}
\operatorname{Ext}_{A_{*}}\left(\mathbb{F}_{p}, H_{*}\left(B P \wedge L_{n}\right)\right) & \cong \operatorname{Ext}_{A_{*}}\left(\mathbb{F}_{p}, A_{*} \square E_{E_{*}} H_{*}\left(L_{n}\right)\right) \\
& \cong \operatorname{Ext}_{E_{*}}\left(\mathbb{F}_{p}, H_{*}\left(L_{n}\right)\right)
\end{aligned}
$$

Lemma 3.5 gives the coaction $\tilde{\psi}: H_{*}\left(L_{n}\right) \rightarrow E_{*} \otimes H_{*}\left(L_{*}\right)$ as follows

$$
\begin{array}{r}
\tilde{\psi}\left(y_{i}\right)=1 \otimes y_{i}, \tilde{\psi}\left(z_{i}\right)=1 \otimes z_{i}+\tau_{0} \otimes y_{i}-\tau_{1} \otimes y_{i-1} \\
\left(\text { Put } y_{-1}=0\right)
\end{array}
$$

Hence we may regard $H_{*}\left(L_{n}\right)$ as an $E\left(\tau_{0}, \tau_{1}\right)$-comodule. Put $E^{\prime}=E\left(\tau_{2}, \tau_{3}, \ldots\right)$, then $E_{*}=E\left(\tau_{0}, \tau_{1}\right) \otimes E^{\prime}$. By the Künneth theorem, it is easy to see that the external pairing

$$
\operatorname{Ext}_{E\left(\tau_{0}, \tau_{1}\right)}\left(\mathbb{F}_{p}, H_{*}\left(L_{n}\right)\right) \otimes \operatorname{Ext}_{E}\left(\mathbb{F}_{p}, \mathbb{F}_{p}\right) \rightarrow \operatorname{Ext}_{E_{*}}\left(\mathbb{F}_{p}, H_{*}\left(L_{n}\right)\right)
$$

is an isomorphism. Since each $\tau_{i}$ is primitive in $E_{*}$, we have

$$
\operatorname{Ext}_{E^{\prime}}\left(\mathbb{F}_{p}, \mathbb{F}_{p}\right)=\mathbb{F}_{p}\left[v_{2}, v_{3}, \ldots, v_{i}, \ldots\right], \text { bideg } v_{i}=\left(1,2 p^{i}-1\right)
$$

$v_{i}$ is represented by $\left[\tau_{i}\right]$ in the cobar complex.

To compute $\operatorname{Ext}_{E\left(\tau_{0}, \tau_{1}\right)}\left(\mathbb{F}_{p}, H_{*}\left(L_{n}\right)\right)$, we apply the Cartan-Eilenberg spectral sequence $([18], p .331)$ to an extension of Hopf algebras $E\left(\tau_{1}\right) \rightarrow E\left(\tau_{0}, \tau_{1}\right)$ $\rightarrow E\left(\tau_{0}\right)$. Clearly, $H_{*}\left(L_{n}\right)$ is a free $E\left(\tau_{0}\right)$-comodule with basis in degrees $2 i(p$ $-1)(i=0,1, \ldots, p-1)$. Therefore

$$
\operatorname{Ext}_{E\left(\tau_{0}\right)}^{s, t}\left(\mathbb{F}_{p}, H_{*}\left(L_{n}\right)\right) \cong \begin{cases}\mathbb{F}_{p} & s=0, t=2 i(p-1) \quad 0 \leqq i \leqq p-1 \\ 0 & \text { otherwise }\end{cases}
$$

By dimensional reason, coaction $\operatorname{Ext}_{E\left(\tau_{0}\right)}\left(\mathbb{F}_{p}, H_{*}\left(L_{n}\right)\right) \rightarrow E\left(\tau_{1}\right) \otimes \operatorname{Ext}_{E\left(\tau_{0}\right)}\left(\mathbb{F}_{p}\right.$, $\left.H_{*}\left(L_{n}\right)\right)$ is trivial. Thus the $E_{2}$-term of the Cartan-Eilenberg spectral sequence is given by 


$$
\begin{aligned}
E_{2}^{s, t} & =\operatorname{Ext}_{E\left(\tau_{1}\right)}^{s}\left(\mathbb{F}_{p}, \operatorname{Ext}_{E\left(\tau_{0}\right)}^{t}\left(\mathbb{F}_{p}, H_{*}\left(L_{n}\right)\right)\right) \\
& \cong \operatorname{Ext}_{E\left(\tau_{0}\right)}^{t}\left(\mathbb{F}_{p}, H_{*}\left(L_{n}\right)\right) \otimes \operatorname{Ext}_{E\left(\tau_{1}\right)}^{s}\left(\mathbb{F}_{p}, \mathbb{F}_{p}\right)
\end{aligned}
$$

Therefore $E_{2}^{s, t}=0$ if $t \neq 0$ and we see that this spectral sequence collapses. We also denote by $y_{i}$ the element of $\operatorname{Ext}_{E\left(\tau_{0}, \tau_{1}\right)}^{0,2 i(p-1)}\left(\mathbb{F}_{p}, H_{*}\left(L_{n}\right)\right)$ represented by $y_{i} \in H_{2 i(p-1)}\left(L_{n}\right)$ in the cobar complex. As in (3.9), we have

$$
\operatorname{Ext}_{E\left(\tau_{1}\right)}\left(\mathbb{F}_{p}, \mathbb{F}_{p}\right)=\mathbb{F}_{p}\left[v_{1}\right], \operatorname{Ext}_{E\left(\tau_{0}, \tau_{1}\right)}\left(\mathbb{F}_{p}, \mathbb{F}_{p}\right)=\mathbb{F}_{p}\left[v_{0}, v_{1}\right]
$$

Hence the $E_{\infty}$-term is given by

$$
E_{\infty}=\mathbb{F}_{p}\left\{y_{0}, y_{1}, \ldots, y_{p-1}\right\} \otimes \mathbb{F}_{p}\left[v_{1}\right]
$$

The second formula of (3.7) settles the extension problem on the $\operatorname{Ext}_{E\left(\tau_{0}, \tau_{1}\right)}\left(\mathbb{F}_{p}, \mathbb{F}_{p}\right)$-module structure of $\operatorname{Ext}_{E\left(\tau_{0}, \tau_{1}\right)}\left(\mathbb{F}_{p}, H_{*}\left(L_{n}\right)\right)$, namely, relations $v_{0} y_{i}=v_{1} y_{i-1}(i=0,1, \ldots, p-1)$ hold in $\operatorname{Ext}_{E\left(\tau_{0}, \tau_{1}\right)}\left(\mathbb{F}_{p}, H_{*}\left(L_{n}\right)\right)$. By iso-morphisms (3.6) and (3.8), we see that the $E_{2}$-term of the Adams spectral sequence is generated by $y_{i} \in \operatorname{Ext}_{A_{*}}^{0,2 i(p-1)}\left(\mathbb{F}_{p}, H_{*}\left(B P \wedge L_{n}\right)\right) \quad$ over $\operatorname{Ext}_{A_{*}}\left(\mathbb{F}_{p}, H_{*}(B P)\right)$ $=\mathbb{F}_{p}\left[v_{0}, v_{1}, \ldots, v_{i}, \ldots\right]$ with relations $v_{0} y_{i}=v_{1} y_{i-1}\left(i=0,1, \ldots, p-1 ; y_{-1}\right.$ $=0)$. Since $E_{2}^{s, t}=0$ if $t-s$ is odd, the Adams spectral sequence collapses. Thus we have shown

Lemma 3.14。 $B P_{*}\left(L_{n}\right)$ is generated by $y_{i} \in B P_{2 i(p-1)}\left(L_{n}\right)(i=0,1, \ldots, p-1)$ over $B P_{*}$ with relations $p y_{0}=0, p y_{i}=v_{1} y_{i-1}(i=1,2, \ldots, p-1)$ and we can choose $y_{i}$ such that it is mapped to $y_{i} \in H_{*}\left(L_{n}\right)$ by the Thom map $B P_{*}\left(L_{n}\right)$ $\rightarrow H_{*}\left(L_{n}\right)$.

Remark 3.15. In $B P_{*}\left(L_{n}\right)$, equality $p B P_{t}\left(L_{n}\right)=v_{1} B P_{t-2(p-1)}\left(L_{n}\right)$ holds for $t$ $<2 p(p-1)$ and the above $y_{i}^{\prime}$ s are determined uniquely modulo $p B P_{*}\left(L_{n}\right)$ so that relations $p y_{i}=v_{1} y_{i-1}(0 \leqq i \leqq p-1)$ hold. These relations imply that $p^{i+1} y_{i}$ $=0$ (but $p^{i} y_{i} \neq 0$ ).

Recall that the Thom map $B P_{*} B P \rightarrow A_{*}$ sends $t_{i}$ to the conjugate of $\xi_{i}$ ([24]). It follows from Lemma 3.5 and the relations that there exists $\lambda_{i, j} \in \mathbb{Z}_{(p)}$ such that the coaction $\psi: B P_{*}\left(L_{n}\right) \rightarrow B P_{*} B P \otimes_{B P_{*}} B P_{*}\left(L_{n}\right)$ is of the following form

$$
\psi\left(y_{i}\right)=\sum_{j=0}^{i}\left\{(-1)^{j}\left(\begin{array}{c}
n+i+1 \\
j
\end{array}\right)+p \lambda_{i, j}\right\} t_{1}^{j} \otimes y_{i-j}
$$

We note that, since $p^{i+1} y_{i}=0$, we may regard $\lambda_{i, j}$ as an element of $\mathbb{Z} / p^{i-j}$, and that each $\lambda_{i, j} \in \mathbb{Z} / p^{i-j}$ does not depend on the choice of generators $y_{0}, y_{1}, \ldots$, $y_{p-1}$ which have the properties of Lemma 3.14. We do not need explicit values of $\lambda_{i, j}$ 's for our purpose, so we only remark that $\lambda_{i, 0} \equiv 0$ modulo $p^{i}$ by the existence of counit, and that $\lambda_{p-1,1}$ determines every other $\lambda_{i, j}$ by the coassociativity and the $B P_{*}$-linearity of $\psi$. 
Define a filtration of $B P_{*}\left(L_{n}\right)$ by putting $F_{t}=B P_{*}\left\{y_{0}, y_{1}, \ldots, y_{t}\right\}$, then 0 $=F_{-1} \subset F_{0} \subset F_{1} \subset \ldots \subset F_{p-1}=B P_{*}\left(L_{n}\right)$ and the associated graded comodule $E_{0}^{t} B P_{*}\left(L_{n}\right)$ is isomorphic to $B P_{*}\left(\Sigma^{2 t(p-1)} M_{p}\right)$ as a $B P_{*} B P$-comodule where $M_{p}$ is the mod $p$ Moore spectrum. We also filter the cobar complex $\Omega^{*}\left(B P_{*}\left(L_{n}\right)\right)$ by $F_{s, t}=B P_{*} B P^{\otimes s} \otimes{ }_{B P_{*}} F_{t}$. Consider the spectral sequence associated with this filtration. Then, we have $E_{1}^{s, t, u}=\operatorname{Ext}_{B P_{*} B P}^{s, u}\left(B P_{*}, E_{0}^{t} B P_{*}\left(L_{n}\right)\right)$ and $d_{r}: E_{r}^{s, t, u} \rightarrow$ $E_{r}^{s+1, t-r, u}$. Note that this spectral sequence has an $\operatorname{Ext}_{B P_{*} B P}\left(B P_{*}, B P_{*}\right)$-module structure. Let $\alpha_{1} \in \operatorname{Ext}_{B P_{*} B P}^{1,2(p-1)}\left(B P_{*}, B P_{*}\right)$ be the element represented by $\left[t_{1}\right]$ in the cobar complex. The $E_{1}$-term is easily obtained by the following results of Miller-Ravenel-Wilson.

Theorem 3.17. ([15], see also [18] p.157) $\operatorname{Ext}_{B P_{*} B P}^{0, *}\left(B P_{*}, B P_{*}\left(M_{P}\right)\right)=\mathbb{F}_{p}\left[v_{1}\right]$, $\operatorname{Ext}_{B P_{*} B P}^{1, *}\left(B P_{*}, B P_{*}\left(M_{p}\right)\right)=\mathbf{F}_{p}\left[v_{1}\right] \cdot \alpha_{1}$ for $*<2 p(p-1)$, and $\operatorname{Ext}_{B P_{*} B P}^{s, u}\left(B P_{*}, B P_{*}\left(M_{p}\right)\right)$ $=0$ for $s \geqq 2$ and $u-s<2 p(p-1)-2$.

Lemma 3.18. $E_{1}^{0, t, u} \cong\left\{\begin{array}{c}\mathbb{Z} / p u=2 j(p-1), j \geqq t \\ 0 \text { otherwise }\end{array}\right.$

$$
E_{1}^{1, t, u} \cong\left\{\begin{array}{c}
\mathbf{Z} / p \quad u=2 j(p-1), t+1 \leqq j<p+t \\
0 \quad \text { otherwise for } u<2(p+t)(p-1)
\end{array}\right.
$$

$E_{1}^{s, t, u}=0$ for $s \geqq 2, u-s<2(t+p)(p-1)-2$. Moreover, $E_{1}^{0, t, 2(i+t)(p-1)}$ is generated by $v_{1}^{i} y_{t}$ and $E_{1}^{1, t, 2(i+t)(p-1)}$ is generated by $\alpha_{1} v_{1}^{i-1} y_{t}(i<p)$.

The first differential is given by (3.16).

$$
d_{1}\left(v_{1}^{i} y_{t}\right)=(n+i+t+1) \alpha_{1} v_{1}^{i} y_{t-1} \text { for } i+t<p .
$$

Put $-n-1=p q_{n}+r_{n}\left(0 \leqq r_{n}<p\right)$, then $d_{1}\left(v_{1}^{i} y_{t}\right)=(n+i+t+1) \alpha_{1} v_{1}^{i} y_{t-1}$ $\neq 0$ if $i+t \neq r_{n}, i+t<p$ and $t>0 . \quad d_{1}\left(v_{1}^{i} y_{t}\right)=0$ if $i+t=r_{n}$ or $t=0$. This implies

Lemma 3.20. $E_{2}^{0, t, u}=0$ if $u \neq 2 r_{n}(p-1), u<2 p(p-1)$ and $0<t<p$. $E_{2}^{1, t, u}=0$ if $u \neq 2 r_{n}(p-1) u<2 p(p-1)$ and $0 \leqq t<p-1$. On the other hand, $E_{2}^{0,0,2 i(p-1)} \cong \mathbb{Z} / p$ for $0 \leqq i<p$, and $E_{2}^{1, r_{n}-i, 2 r_{n}(p-1)} \cong \mathbb{Z} / p$ for $1 \leqq i \leqq r_{n}$ if $r_{n}>0$.

Define a filtration on $\operatorname{Ext}_{B P_{*} B P}^{s, u}\left(B P_{*}, B P_{*}\left(L_{n}\right)\right)$ by $F^{s, t, u}=\operatorname{Im}$ $\left[\operatorname{Ext}_{B P_{*} B P}^{s, u}\left(B P_{*}, F_{t}\right) \rightarrow \operatorname{Ext}_{B P_{*} B P}^{s, u}\left(B P_{*}, B P_{*}\left(L_{n}\right)\right)\right]$. Then $0=F^{s,-1, u} \subset F^{s, 0, u} \subset \ldots$ $\subset F^{s, t, u} \subset \ldots \subset F^{s, p-1, u}=\operatorname{Ext}_{B P_{*} B P}^{s, u}\left(B P_{*}, B P_{*}\left(L_{n}\right)\right)$ and $F^{s, t, u} / F^{s, t-1, u} \cong E_{\infty}^{s, t, u}$.

Lemma 3.21. For $j<2 p(p-1)-2, \pi_{j}^{S}\left(L_{n}\right) \neq 0$ if and only if one of the following conditions is satisfied;

i) $j=2 i(p-1)$ for $0 \leqq i<p$.

ii) $j=2 r_{n}(p-1)-1$ and $r_{n}>0$.

Proof. Consider the Adams-Novikov spectral sequence $E_{2}^{s, u}\left(L_{n}\right)=$ 
$\operatorname{Ext}_{B P_{*} B P}^{s, u}\left(B P_{*}, B P_{*}\left(L_{n}\right)\right) \Rightarrow \pi_{t-s}^{s}\left(L_{n}\right) . \quad$ By Lemma 3.18 and Lemma 3.20, $E_{\infty}^{0, t, u}=0$ if $u \neq 0$ modulo $2(p-1)$, and $E_{\infty}^{1, t, u}=0$, if $u=0$ or $u \neq 2 r_{n}(p-1), u<2 p(p-1)$. Hence we have $\operatorname{Ext}_{B P * B P}^{0, u}\left(B P_{*}, B P_{*}\left(L_{n}\right)\right)=0$ if $u$ 三 0 modulo $2(p-1)$, and $\operatorname{Ext}_{B P_{*} B P}^{1, u}\left(B P_{*}, B P_{*}\left(L_{n}\right)\right)=0$ if $u=0$ or $u \neq 2 r_{n}(p-1), u<2 p(p-1)$. Since $E_{\infty}^{s, t, u}=E_{1}^{s, t, u}=0 \quad$ for $\quad s \geqq 2, \quad u-s<2 p(p-1)-2, \quad$ we also have $\operatorname{Ext}_{B P_{*} B P}^{s, u}\left(B P_{*}, B P_{*}\left(L_{n}\right)\right)=0$ for $s \geqq 2, u-s<2 p(p-1)-2$. It follows that $\pi_{j}^{S}\left(L_{n}\right)=0(j<2 p(p-1)-2)$ if neither i) nor ii) is satisfied. Conversely, since $E_{\infty}^{0,0,2 i(p-1)}=E_{2}^{0,0,2 i(p-1)} \neq 0$ for $0 \leqq i<p$, we see that $\operatorname{Ext}_{B P * B P}^{0,2 i(p-1)}$ $\left(B P_{*}, B P_{*}\left(L_{n}\right)\right) \neq 0$ for $0 \leqq i<p$. By Lemma 3.18 and Lemma 3.20, $E_{2}^{1, r_{n}-1,2 r_{n}(p-1)} \neq 0$ if $r_{n} \neq 0, E_{2}^{0, t, 2 r_{n}(p-1)}=0$ if $t>r_{n}$, and $E_{2}^{2, t, u}=0$ if $u<2 p(p$ $-1)$. Therefore elements of $E_{2}^{1, r_{n}-1,2 r_{n}(p-1)}$ survive and $E_{\infty}^{1, r_{n}-1,2 r_{n}(p-1)} \neq 0$, thus $\operatorname{Ext}_{B P_{*} B P}^{1,2 r_{n}(p-1)}\left(B P_{*}, B P_{*}\left(L_{n}\right)\right) \neq 0$ if $r_{n} \neq 0$. Recalling that $\operatorname{Ext}_{B P_{*} B P}^{s, u}\left(B P_{*}, B P_{*}\left(L_{n}\right)\right)=0$ for $s \geqq 2, u-s<2 p(p-1)-2$, it follows that $E_{\infty}^{0,2 i(p-1)}\left(L_{n}\right)=E_{2}^{0,2 i(p-1)}\left(L_{n}\right) \neq 0$ for $0 \leqq i<p$ and $E_{\infty}^{1,2 r_{n}(p-1)}\left(L_{n}\right)$ $=E_{2}^{1,2 r_{n}(p-1)}\left(L_{n}\right) \neq 0$ if $r_{n} \neq 0$. This completes the proof.

Theorem 3.22。 For $i<2 p n+2 p^{2}-4$ and $n \geqq p-1, \pi_{i}\left(Q S^{2 n+1}, S^{2 n+1}\right) \neq 0$ if and only if one of the following conditions is satisfied;

i) $i=2 p n+2 j(p-1)$ for $1 \leqq j \leqq p$.

ii) $i=2\left(r_{n}+1\right)(p-1)+2 p n-1$ and $r_{n}>0$.

Proof. $\pi_{i}\left(Q S^{2 n+1}, S^{2 n+1}\right)$ is isomorphic to $\pi_{i-1}(F(2 n+1))$ and, by Corollary $3.2, \pi_{i-1}(F(2 n+1))$ is isomorphic to the stable homotopy group $\pi_{i-1}^{S}(F(2 n+1))$ if $i<4 p n+4 p-6$. By the definition of $L_{n}, \pi_{i-1}^{S}(F(2 n+1)) \cong \pi_{i-2 p n-2 p+2}^{S}\left(L_{n}\right)$ for $i<2 p n+2 p^{2}-4$. Note that $2 p n+2 p^{2}-4 \leqq 4 p n+4 p-6$ since $n \geqq p$ -1 , and we have an isomorphism $\pi_{i}\left(Q S^{2 n+1}, S^{2 n+1}\right) \cong \pi_{i-2 p n-2 p+2}^{S}\left(L_{n}\right)$ for $i$ $<2 p n+2 p^{2}-4$. Then, the assertion follows from the previous lemma.

In the case $n<p-1$, we put $m=[(n p+p-2) /(p-1)]$ and define a spectrum $L_{n}^{\prime}$ to be $\Sigma^{-2 n p-2 p+3} \tilde{F}(2 n+1)^{4 n p+4 p-6}$ instead of $L_{n}$. Then $\left\{y_{0}, z_{0}, y_{1}, z_{1}, \ldots, y_{m}, z_{m}\right\}$ is a basis of $H_{*}\left(L_{n}^{\prime}\right)$, and as in Lemma $3.14, B P_{*}\left(L_{n}^{\prime}\right)$ is generated by $y_{i} \in B P_{*}\left(L_{n}^{\prime}\right)$, we can compute $\operatorname{Ext}_{B P_{*} B P}\left(B P_{*}, B P\left(L_{n}^{\prime}\right)\right)$ in the same way. Thus we have

Theorem 3.23. For $i<4 p n+4 p-6$ and $n<p-1, \pi_{i}\left(Q S^{2 n+1}, S^{2 n+1}\right) \neq 0$ if and only if one of the following conditions is satisfied;

i) $i=2 p n+2 j(p-1)$ for $1 \leqq j \leqq[(n p+p-2) /(p-1)]$.

ii) $i=2\left(r_{n}+1\right)(p-1)+2 p n-1$ and $r_{n}>0$.

We also need to know whether $\pi_{i}\left(Q S^{2 n+1}, S^{2 n+1}\right)$ is zero or not in the low dimensional unstable range. The following result is due to Toda (see also [2]).

Theorem 3.24. ([22]). For $1 \leqq n<p-1$,

$$
\pi_{i}\left(Q S^{2 n+1}, S^{2 n+1}\right) \cong\left\{\begin{array}{lll}
\mathbb{Z} / p & i=2 p n+2 j(p-1), & 1 \leqq j<p-n \\
0 & \text { otherwise for } & i<2 n+2 p(p-1)
\end{array}\right.
$$


Summarizing Theorem 3.22, 3.23 and 3.24,

(3.25) For $i<\min \left\{2 p n+2 p^{2}-4, \quad \max \{4 p n+4 p-6, \quad 2 n+2 p(p-1)\}\right\}$, $\pi_{i}\left(Q S^{2 n+1}, S^{2 n+1}\right) \neq 0$ if and only if one of the following conditions is satisfied.

i) $i=2 p n+2 j(p-1)$ for $j \geqq 1$.

ii) $i=2\left(r_{n}+1\right)(p-1)+2 p n-1$ and $r_{n}>0$, where $-n-1=p q_{n}+r_{n}, 0 \leqq r_{n}$ $<p$.

Remark 3.26. For an integer $i$ which satisfies the condition i) above, the Hurewicz map $\pi_{i-1}(F(2 n+1)) \rightarrow P B P_{i-1}(F(2 n+1))$ is an isomorphism, where $P B P_{*}(X)$ is the set of primitive elements $\left\{x \in B P_{*}(X) \mid \psi(x)=1 \otimes x\right\}$.

\section{$\S 4$. The $p$-Regularity for an Odd Prime $p$}

As in the previous section, $p$ is a fixed odd prime and all spaces and groups are localized at $p$ throughout this section. First, we note the following fact which is easily obtained by Corollary 1.2.

Proposition 4.1. $W_{n+k, k}$ is not $p$-regular if $k>p$, or $k=p$ and $p \nmid n . \quad X_{n+k, k}$ is not p-regular if $k>(p+1) / 2$, or $k=(p+1) / 2$ and $p \nmid 2 n+1$.

We recall the conditions $(\mathrm{C})$ and $(\mathrm{H})$ in Section 1 .

(C) $k \leqq p-1$, or $k=p$ and $p \mid n$. (H) $k \leqq(p-1) / 2$, or $k=(p+1) / 2$ and $p \mid 2 n+1$.

The following result of James is the starting point of our argument.

Theorem 4.2. ([8]) Corollary 7. 11) $Q_{n+k, k}(\mathbb{F})$ is a stable retract of $O_{n+k, k}(\mathbb{F})$, where $O_{n+k, k}(\mathbf{F})$ is $V_{n+k, k}, W_{n+k, k}$ or $X_{n+k, k}$ for $\mathbf{F}=\mathbf{R}, \mathbf{C}$ or $\mathbf{H}$ respectively.

The combination of the above theorem and Theorem 1.8 implies

Lemma 4.3. i) If (C) is satisfied, there exists a map $f:\left(W_{n+k, k}, S^{2 n+1}\right)$ $\rightarrow\left(Q S^{2 n+1}, S^{2 n+1}\right)$ such that $f \mid S^{2 n+1}: S^{2 n+1} \rightarrow S^{2 n+1}$ is degree 1 .

ii) If $(\mathrm{H})$ is satisfied, there exists a map $g:\left(X_{n+k, k}, S^{4 n+3}\right) \rightarrow\left(Q S^{4 n+3}, S^{4 n+3}\right)$ such that $g \mid S^{4 n+3}: S^{4 n+3} \rightarrow S^{4 n+3}$ is degree 1 .

Proof. By Theorem 1.8, there exist maps $g^{\prime}: Q_{n+k, k}(\mathbb{C}) \rightarrow S^{2 n+1}$ and $g^{\prime}$ : $Q_{n+k, k}(\mathrm{H}) \rightarrow S^{4 n+3}$ such that the compositions $S^{2 n+1} \hookrightarrow Q_{n+k, k}(\mathbb{C}) \stackrel{f^{\prime}}{\longrightarrow} S^{2 n+1}$ and $S^{4 n+3} \hookrightarrow Q_{n+k, k}(\mathbf{H}) \stackrel{g^{\prime}}{\longrightarrow} S^{4 n+3}$ are degree 1. Let $f^{\prime \prime}: \Sigma^{\infty} W_{n+k, k} \rightarrow \Sigma^{\infty} Q_{n+k, k}(\mathbf{C})$ and $g^{\prime \prime}: \Sigma^{\infty} X_{n+k, k} \rightarrow \Sigma^{\infty} Q_{n+k, k}(\mathbf{H})$ be stable retractions. Define $f$ and $g$ to be the adjoints of stable maps $\Sigma^{\infty} f^{\prime} \circ f^{\prime \prime}: \Sigma^{\infty} W_{n+k, k} \rightarrow \Sigma^{\infty} S^{2 n+1}$ and $\Sigma^{\infty} g^{\prime} \circ g^{\prime \prime}: \Sigma^{\infty} X_{n+k, k}$ $\rightarrow \Sigma^{\infty} S^{4 n+3}$, respectively. Then $f$ and $g$ satisfy the conditions.

We define sets of integers as follows 
(4.4) $C_{n+k, k}(\mathbb{F})=\left\{i \in \mathbb{Z} \mid i=\sum_{j=1}^{k} \varepsilon_{j}(d n+d j-1), \varepsilon=0\right.$ or 1$\}$, where $d=2$ if $\mathbb{F}$ $=\mathbb{C}$ and $d=4$ if $\mathbb{F}=\mathbb{H}$.

(4.5) $\Pi(n ; p)=\left\{i \in \mathbb{Z} \mid i=2 p n+2 j(p-1)\right.$ for $j \geqq 1$, or $i=2\left(r_{n}+1\right)(p-1)+2 p n$ -1 if $\left.r_{n}>0\right\}$ where $r_{n}$ is defined by $-n-1=p q_{n}+r_{n}, 0 \leqq r_{n}<p$ (See Introduction.).

Note that $C_{n+k, k}(\mathbb{C})=\left\{i \in \mathbb{Z} \mid H^{i}\left(W_{n+k, k}\right) \neq 0\right\} \quad$ and $\quad C_{n+k, k}(\mathbb{H})$ $=\left\{i \in \mathbb{Z} \mid H^{i}\left(X_{n+k, k}\right) \neq 0\right\}$. We also note that obstructions to deform $f($ resp. $g)$ to $S^{2 n+1}\left(\operatorname{resp} . S^{4 n+3}\right)$ lie in $H^{i}\left(W_{n+k, k}, S^{2 n+1} ; \pi_{i}\left(Q S^{2 n+1}, S^{2 n+1}\right)\right)$ (resp. $\mathrm{H}^{i}\left(X_{n+k, k}\right.$, $\left.\left.S^{4 n+3} ; \pi_{i}\left(Q S^{4 n+3}, S^{4 n+3}\right)\right)\right)$.

Theorem 4.6. i) Assume $k \leqq p-1$, or $k=p$ and $p \mid n$, then $W_{n+k, k}$ is homotopy equivalent to $S^{2 n+1} \times W_{n+k, k-1}$ if $C_{n+k, k}(\mathbb{C}) \cap \Pi(n ; p)$ is empty. ii) Assume $k \leqq(p-1) / 2$, or $k=(p+1) / 2$ and $p \mid 2 n+1$, then $X_{n+k, k}$ is homotopy equivalent to $S^{4 n+3} \times X_{n+k, k-1}$ if $C_{n+k, k}(\mathbb{H}) \cap \mathbb{\Pi}(2 n+1 ; p)$ is empty.

Proof. First note that $\operatorname{dim} W_{n+k, k}=\max C_{n+k, k}(\mathbb{C})=2 n k+k^{2}<\min \{2 p n$ $\left.+2 p^{2}-4, \max \{4 p n+4 p-6,2 n+2 p(p-1)\}\right\}$ if $k \leqq p-1$, or $k=p$ and $p \mid n$. Hence, by (3.25), the condition of i) is equivalent to vanishing of $H^{i}\left(W_{n+k, k}\right.$, $\left.S^{2 n+1} ; \pi_{i}\left(Q S^{2 n+1}, S^{2 n+1}\right)\right)$ for any $i \in \mathbb{Z}$. Therefore there exists a left homotopy inverse of the inclusion $S^{2 n+1} \hookrightarrow W_{n+k, k}$, and fibration $S^{2 n+1} \rightarrow W_{n+k, k} \rightarrow W_{n+k, k-1}$ is fiber homotopy equivalent to the product fibration $S^{2 n+1} \rightarrow S^{2 n+1} \times W_{n+k, k-1}$ $\rightarrow W_{n+k, k-1}$ ([8], Corollary 4.3). Proof of ii) is similar.

If $n \geqq\left(k^{2}-2 p+3\right) /(2 p-2 k)(k \leqq p-1), \operatorname{dim} W_{n+k, k} \leqq 2 p n+2 p-3$. Hence $C_{n+k, k-j}(\mathbb{C}) \cap \Pi(n+j ; p)$ is empty for $j=0,1, \ldots, k-1$. And if $m \geqq(p-1) / 2$, it is easy to verify that $C_{m p+p, p}(\mathbb{C}) \cap \Pi(m p ; p)$ is empty. Similarly, if $n \geqq\left(2 k^{2}+k\right.$ $-4 p+3) /(4 p-4 k)(k \leqq(p-1) / 2), C_{n+k, k-j}(H) \cap \mathbb{H}(2 n+2 j+1 ; p)$ is empty for $j=0,1, \ldots, k-1$, and if $p \mid 2 n+1$ (hence $n \geqq(p-1) / 2), C_{n+(p+1) / 2,(p+1) / 2}(\mathbb{H})$ $\cap \Pi(2 n+1 ; p)$ is empty. Thus the preceding theorem implies

Theorem 4.7. i) For $k \leqq p-1, W_{n+k, k}$ is p-regular if $\bigcup_{j=0}^{k-1}\left(C_{n+k, k-j}(\mathbb{C})\right.$ $\bigcap \Pi(n+j ; p))$ is empty. In particular, it is p-regular if $n \geqq\left(k^{2}-2 p++3\right) /(2 p$ $-2 k)$. And $W_{m p+p, p}$ is $p$-regular if $m \geqq(p-1) / 2$.

ii) For $k \leqq(p-1) / 2, X_{n+k, k}$ is p-regular if $\bigcup_{j=0}^{k-1}\left(C_{n+k, k-j}(\mathbb{H}) \cap \mathbb{I}(2 n+2 j+1\right.$; $\left.p)\right)$ is empty. In particular, it is p-regular if $n \geqq\left(2 k^{2}+k-4 p+3\right) /(4 p-4 k)$. And $X_{n+(p+1) / 2,(p+1) / 2}$ is $p$-regular if $p \mid 2 n+1$.

The fact that $\pi_{2 n+1}(B U(n))$ (resp. $\left.\pi_{4 n+3}(B S p(n))\right)$ is a trivial group if $n<p$ (resp. $n<(p-1) / 2$ ) easily implies the $p$-regularity of $W_{n+k, k}$ (resp. $X_{n+k, k}$ ) for $n$ $+k \leqq p$ (resp. $n+k \leqq(p-1) / 2)$.

Proposition 4.8 ([6]). $W_{n+k, k}$ is p-regular if $n+k \leqq p$, and $X_{n+k, k}$ is $p$ regular if $n+k \leqq(p-1) / 2$. 
Proof. Consider the fiber bundle $W_{n+k-1, k-1} \rightarrow W_{n+k, k} \rightarrow S^{2 n+2 k-1}$ which is associated with the principal bundle $U(n+k-1) \rightarrow U(n+k) \rightarrow S^{2 n+2 k-1}$. Then the classifying map is an element of $\pi_{2 n+2 k-1}(B U(n+k-1))$. If $n+k \leqq p$, $\pi_{2 n+2 k-1}(B U(n+k-1)) \cong \mathbf{Z}_{(p)} /(n+k-1) !=0$. Therefore the above fiber bundle is trivial and $W_{n+k, k}$ is homotopy equivalent to $W_{n+k-1, k-1} \times S^{2 n+2 k-1}$. Inductively, we can show the $p$-regularity of $W_{n+k, k}$. Proof of the second statement is similar.

Remark 4.9. i) It follows from Theorem 4.7 and Proposition 4.8 that $W_{n+k, k}$ is $p$-regular for any $n$ if $k \leqq[(2-\sqrt{2}) p]+1$ and that $X_{n+k, k}$ is $p$-regular for any $n$ if $k \leqq(p-1) / 2$ for $p=3,5,7,11$, and $k \leqq[(3-\sqrt{5}) p / 2]+1$ for $p \geqq 13$.

ii) Since the obstruction group $\pi_{2 p n+2 i(p-1)}\left(Q S^{2 n+1}, S^{2 n+1}\right) \cong \pi_{2 p n+2 i(p-1)-1}$ $(F(2 n+1))(i \geqq 1)$ maps isomorphically onto $P B P_{2 p n+2 i(p-1)-1}(F(2 n+1))$ by the $B P$-Hurewicz map as we noted in Remark 3.26 , we may be able to know whether the obstruction in this dimension vanishes or not, if we can describe the map $f_{*}: B P_{*}\left(W_{n+k, k}\right) \rightarrow B P_{*}\left(Q S^{2 n+1}\right)$ explicitly (for this, $f: W_{n+k, k} \rightarrow Q S^{2 n+1}$ should be constructed explicitly). We also remark that if $n>\left(k^{2}-4 p+4\right) /(4 p-2 k)$, we may assume that the image of $f$ is contained in the $(p+1)$-th May filtration $F_{p+1} C_{\infty} S^{2 n+1}$ of $C_{\infty} S^{2 n+1} \simeq Q S^{2 n+1} \quad([13])$ and $B P_{*}\left(F_{p+1} C_{\infty} S^{2 n+1}\right)$ can be computed in the same way as we computed $B P_{*}\left(L_{n}\right)$.

Examples 4.10. i) For $p=3, W_{n+k, k}$ is 3-regular if and only if $k \leqq 2$, or $k$ $=3$ and $3 \mid n$. Assume that $k \leqq p-1$, or $k=p$ and $p \mid n$. $W_{n+k, k}$ is $p$-regular except for the following undecided cases;

For $p=5,(n, k)=(4,4),(5,5)$

For $p=7,(n, k)=(2,6),(3,6),(6,6),(12,6),(7,7),(14,7)$

For $p=11,(n, k)=(4,8),(i, 9)$ for $3 \leqq i \leqq 10,(i, 10)$ for $2 \leqq i \leqq 12$,

$$
(40,10),(11 i, 11) \text { for } i=1,2,3,4
$$

For $p=13,(n, k)=(5,9),(i, 10)$ for $i=4,5,6,7,(i, 11)$ for $3 \leqq i \leqq 16,(i, 12)$

$$
\text { for } 2 \leqq i \leqq 18,(12 i, 12) \text { for } i=2,3,4,5 \text {, }
$$

$(13 i, 13)$ for $1 \leqq i \leqq 5$.

ii) For $p=3,5,7,11,17, X_{n+k, k}$ is $p$-regular if and only if $k \leqq(p-1) / 2$,

$$
\text { or } k=(p+1) / 2 \text { and } p \mid 2 n+1 \text {. }
$$

Assume that $k \leqq(p-1) / 2$, or $k=(p+1) / 2$ and $p \mid 2 n+1 . \quad X_{n+k, k}$ is $p$-regular except for the following undecided cases.

For $p=13,(n, k)=(1,6)$

For $p=19,(n, k)=(1,9)$

For $p=23,(n, k)=(1,11),(2,11),(3,11)$.

\section{Appendix. The Non-2-Regularity of Real Stiefel Manifolds}

Recall that real quasiprojective space $Q_{n+k, k}(\mathbf{R})$ is in fact real stunted projective space $\mathbf{P}_{n}^{n+k-1}$. To begin with we state the following facts without proofs. 
Lemma $\quad A_{0} \mathbb{1} 。 \widetilde{H}^{*}\left(Q_{n+k, k}(\mathbb{R})\right)=\mathbb{F}_{2}\left\{x_{n}, x_{n+1}, \ldots, x_{n+k-1}\right\}, S q^{i} x_{j}=\left(\begin{array}{l}j \\ i\end{array}\right) x_{i+j}$. Thus the action of the Steenrod operations in trivial if and only if $k=1$, or $k=2$ and $2 \mid n$.

Proposition A.2. The following three conditions are equivalent.

i) $k=1$, or $k=2$ and $2 \mid n$. ii) $Q_{n+k, k}(\mathbb{R})$ is homotopy equivalent to $\vee_{i=1}^{k} S^{n+i-1}$ at prime 2. iii) $\Omega V_{n+k, k}$ is homotopy equivalent to $\prod_{i=1}^{k} \Omega S^{n+i-1}$ at prime 2 . Moreover, if $n$ is even, $Q_{n+2,2}(\mathbb{R})$ is globally homotopy equivalent to $S^{n} \vee S^{n+1}$ and $\Omega V_{n+2,2}$ is globally homotopy equivalent to $\Omega\left(S^{n} \times S^{n+1}\right)$.

Proposition $\mathbb{A}_{\text {. 3. }} V_{n+k, k}$ cannot be 2-regular unless $k=1$, or $k=2$ and $2 \mid n$.

Let $k_{n}: S^{n+1} \rightarrow B S O(n+1)$ be the classifying map of sphere bundle $S^{n}$ $\rightarrow V_{n+2,2} \rightarrow S^{n+1}$, and we also denote by $k_{n} \in \pi_{n}(S O(n+1))$ the element corresponding to $k_{n} \in \pi_{n+1}(B S O(n+1))$ by the natural isomorphism. The following fact is due to Kervaire ([9]).

Lemma A.4. Consider principal bundle $S O(n) \rightarrow S O(n+1) \stackrel{\rho}{\longrightarrow} S^{n}$. Then

$$
\operatorname{Im}\left[\rho_{*}: \pi_{n}(S O(n+1)) \rightarrow \pi_{n}\left(S^{n}\right)\right]= \begin{cases}\pi_{n}\left(S^{n}\right) & \text { for } n=1,3,7 \\ 2 \pi_{n}\left(S^{n}\right) & \text { for odd } n, n \neq 1,3,7 \\ 0 & \text { for even } n\end{cases}
$$

Hence we see that $k_{2}$ and $k_{6}$ are null homotopic. This implies $\times S^{7}$.

Proposition A. 5. There are homeomorphisms $V_{4,2} \cong S^{2} \times S^{3}$ and $V_{8,2} \cong S^{6}$

Note that $k_{n} \in \pi_{n}(S O(n+1))$ is the image of generator $l_{n+1}$ of $\pi_{n+1}\left(S^{n+1}\right)$ by $\Delta: \pi_{n+1}\left(S^{n+1}\right) \rightarrow \pi_{n}(S O(n+1))$.

Lemma A.6. For even $n$, there exists $k_{n}^{\prime} \in \pi_{n}(S O / S O(n))$ which maps to $k_{n} \in \pi_{n}(S O(n+1))$ by composition $\pi_{n+1}(S O / S O(n)) \stackrel{\partial}{\longrightarrow} \pi_{n}(S O(n)) \stackrel{j_{*}}{\longrightarrow} \pi_{n}(S O(n+1))$.

Proof. By Lemma A.4, $j_{*}: \pi_{n}(S O(n)) \rightarrow \pi_{n}(S O(n+1))$ is surjective. Choose $k_{n}^{\prime \prime} \in \pi_{n}(S O(n))$ which maps to $k_{n}$. Since $k_{n}=\Delta\left(l_{n+1}\right)$ is in the kernel of $\pi_{n}(S O(n$ $+1)) \rightarrow \pi_{n}(S O(n+2)) \cong \pi_{n}(S O)$, it is easy to see that $k_{n}^{\prime \prime}$ is in the image of $\partial$ : $\pi_{n+1}(S O / S O(n)) \rightarrow \pi_{n}(S O(n))$.

A proof of the following lemma is similar to that of Lemma 2.16.

Lemma A.7. $\quad \delta_{*}: \pi_{2 n}\left(Q S^{n-1}\right) \rightarrow \pi_{2 n}(F(n))$ is trivial for even $n, n \neq 2,6$.

Lemma A.8. For even $n, n \neq 2,6^{\circ}, k_{n}^{\prime \prime}=\partial\left(k_{n}^{\prime}\right) \in \pi_{n}(S O(n))$ is mapped by $J$ : 
$\pi_{n}(S O(n)) \rightarrow \pi_{n}\left(\Omega_{1}^{n} S^{n}\right)$ to an element which is not in the image of the connecting homomorphism $\delta^{\prime}: \pi_{n+1}\left(S^{n}\right) \rightarrow \pi_{n}\left(\Omega_{1}^{n} S^{n}\right)$ associated with fibration $\Omega_{1}^{n} S^{n} \rightarrow S H(n+1)$ $\rightarrow S^{n}$.

Proof. By the commutativity of (2.12) and the above lemma, composition $\pi_{n+1}(S O / S O(n)) \stackrel{\partial}{\longrightarrow} \pi_{n}(S O(n)) \stackrel{J}{\longrightarrow} \pi_{n}\left(\Omega_{1}^{n} S^{n}\right)$ is injective. Suppose $J\left(k_{n}^{\prime \prime}\right)=\delta^{\prime}(x)$ for some $x \in \pi_{n+1}\left(S^{n}\right)$. Let $j^{\prime}: S^{n} \rightarrow S O / S O(n)$ be the canonical inclusion, then $\Delta$ : $\pi_{n+1}\left(S^{n}\right) \rightarrow \pi_{n}(S O(n))$ factors as $\pi_{n+1}\left(S^{n}\right) \stackrel{j_{*}^{\prime}}{\longrightarrow} \pi_{n+1}(S O / S O(n)) \stackrel{\partial}{\longrightarrow} \pi_{n}(S O(n))$. Also $\delta^{\prime}$ factors as $J \circ \Delta=J \circ \partial \circ j_{*}$. Therefore $J \circ \partial\left(k_{n}^{\prime}\right)=J\left(k_{n}^{\prime \prime}\right)=\delta^{\prime}(x)=J \circ \partial \circ j_{*}^{\prime}(x)$ and we have $k_{n}^{\prime}=j_{*}^{\prime}(x)$ which maps to zero by composition $\pi_{n+1}(S O / S O(n))$ $\rightarrow \pi_{n+1}(S O / S O(n+1)) \stackrel{\partial}{\longrightarrow} \pi_{n}(S O(n+1))$. This contradicts the choice of $k_{n}^{\prime}$ since $k_{n}$ is not zero.

Theorem A.9. Except for the cases $n=0,2,6, V_{n+2,2}$ is not 2-regular.

Proof. By the preceding lemma, $k_{n} \in \pi_{n}(S O(n+1))$ maps to a non-zero element by $J_{H^{*}}: \pi_{n}(S O(n+1)) \rightarrow \pi_{n}(H(n+1))$. Then the result follows from Theorem 2.9 and Proposition 2.10.

An analogue of Corollary 2.18 holds.

Corollary A.10. The attaching map of the top cell of $V_{n+2,2}(n=2 j, j \neq 0,1$, 3) defines a non-trivial element $\xi_{j}$ in the 2-component of $\pi_{4 j}\left(S^{2 j}\right) . \quad \xi_{j}$ is in the kernel of $E^{2}: \pi_{4 j}\left(S^{2 j}\right) \rightarrow \pi_{4 j+2}\left(S^{2 j+2}\right) \cong \pi_{2 j}^{S}\left(S^{0}\right)$ and has order 2 if $j$ is even and at most 4 if $j$ is odd.

Proof. Recall that $Q_{n+2,2}(\mathbb{R})$ is a stable summand of $V_{n+2,2}$ (Theorem 4.2) and $Q_{n+2,2}(\mathbb{R}) \cong S^{n} \vee S^{n+1}$. Hence there exists a map $h:\left(V_{n+2,2}, S^{n}\right) \rightarrow\left(Q S^{n}, S^{n}\right)$ such that $h \mid S^{n}: S^{n} \rightarrow S^{n}$ is degree 1. In fact, $h$ is an extension of composition $Q_{n+2,2}(\mathbb{R}) \stackrel{c}{\longrightarrow} S^{n} \stackrel{i}{\longrightarrow} Q S^{n}$. Thus $i \circ \xi_{j}=i \circ{ }^{\circ} \circ \xi_{j}^{\prime}=0$ where $\xi_{j}^{\prime}: S^{2 n} \rightarrow Q_{n+2,2}(\mathbb{R})$ is the attaching map and $k: Q_{n+2,2}(\mathbb{R}) \rightarrow V_{n+2,2}$ is the inclusion map. This means that $\xi_{j}$ suspends to zero in the stable range. The last assertion follows from the fact that $\left.\pi_{4 j}(F(2 j))\left(\cong \pi_{2 j+1}^{S} \mathbb{P}_{2 j}^{\infty}\right)\right)$ is isomorphic to $\mathbb{Z} / 2 \oplus \mathbb{Z} / 2$ if $j$ is even and to $\mathbb{Z} / 4$ if $j$ is odd.

\section{References}

[1] Adams, J. F., On the non-existence of elements of the Hopf invariant one, Ann. of Math., 12 (1960), 20-104.

[2] Bendersky, M., Some calculations in the unstable Adams-Novikov spectral sequence, Publ. RIMS, Kyoto Univ., 16 (1980), 739-766.

[3] Dold, A. and Lashof, R. K., Principal quasifibration and fibre homotopy equivalence of 
bundles, Ill. J. Math., 3 (1959), 285-305.

[4] Dyer, E. and Lashof, R. K., Homology of iterated loop spaces, Amer. J. Math., 84 (1962), 35-88.

[ 5 ] Harris, B., Some calculations of homotopy groups of symmetric spaces, Trans. Amer. Math. Soc., 106 (1963), 174-184.

[6] Hemmi, Y., Mod p H-spaces and p-regularities, Hiroshima Math. J., 12 (1982), 399-419.

[7] James, I. M., Spaces associated with Stiefel manifolds, Proc. London Math. Soc., 9 (1959), $115-140$.

[8] - The topology of Stiefel manifolds, London Math. Soc. Lecture Notes Series 24, 1976.

[9] Kervaire, M. A., Some nonstable homotopy groups of Lie groups, Ill. J. Math., 4 (1960), 161-169.

[10] Mahowald, M. E., The metastable homotopy of $S^{n}$, Memoirs of Amer. Math. Soc No. 72 (1967).

[11] Mahowald, M. E. and Tangora, M. C., On the secondary operations which detect homotopy classes, Bol. Soc. Mat. Mexicana, 12 (1967), 71-75.

[12] May, J. P., The homology of $E_{\infty}$ spaces, Lecture Notes in Math., 533, Springer-Verlag, 1976, $1-68$.

[13] - The geometry of iterated loop spaces, Lecture Notes in Math., 271, Springer-Verlag, 1972.

[14] Milgram, R. J., Unstable homotopy from the stable point of view, Lecture Notes in Math., 368, Springer-Verlag, 1974.

[15] Miller, H. R., Ravenel, D. C. and Wilson, W. S., Periodic phenomena in the Adams-Novikov spectral sequence, Ann. of Math., 106 (1977), 469-516.

[16] Milnor, J. W., The Steenrod algebra and its dual, Ann. of Math., 67 (1958), 150-171.

[17] Nishida, G., Cohomology operations in iterated loop spaces, Proc. Japan Acad., 44 (1968), 104-109.

[18] Ravenel, D. C., Complex cobordism and stable homotopy groups of spheres, Academic Press, 1986.

[19] Serre, J.-P., Groupes d'homotopie et classes de groupes abéliens, Ann. of Math., 58 (1953), 258-294.

[20] Steenrod, N. E. and Epstein, D. B. A., Cohomology operations, Ann. of Math. Studies No. 50, Princeton, 1962.

[21] Toda, H., Composition methods in homotopy groups of spheres, Ann. of Math. Studies No. 49, Princeton, 1962.

[22] - On iterated suspension I, J. Math. Kyoto Univ., 5 (1965), 89-142.

[23] Whitehead, G. W., A generalization of Hopf invariant, Ann. of Math., 51 (1950), 192-237.

[24] Zahler, R. S., The Adams-Novikov spectral sequence for the spheres, Ann. of Math., 96 (1972), 480-504. 